\title{
Separation of Domain Contacts Is Required for Heterotetrameric Assembly of Functional NMDA Receptors
}

\author{
Anthony N. Farina, ${ }^{1 \star}$ Katherine Y. Blain, ${ }^{2 \star}$ Tomohiko Maruo, ${ }^{1}$ Witek Kwiatkowski, ${ }^{2}$ Senyon Choe, ${ }^{2}$ and \\ Terunaga Nakagawa ${ }^{1}$ \\ ${ }^{1}$ Department of Chemistry and Biochemistry, University of California, San Diego, La Jolla, California 92093 and ${ }^{2}$ Structural Biology Laboratory, Salk \\ Institute, La Jolla, California 92037
}

\begin{abstract}
The precise knowledge of the subunit assembly process of NMDA receptors (NMDA-Rs) is essential to understand the receptor architecture and underlying mechanism of channel function. Because NMDA-Rs are obligatory heterotetramers requiring the GluN1 subunit, it is critical to investigate how GluN1 and GluN2 type subunits coassemble into tetramers. By combining approaches in cell biology, biochemistry, single particle electron microscopy, and x-ray crystallography, we report the mechanisms and phenotypes of mutant GluN1 subunits that are defective in receptor maturation. The T110A mutation in the N-terminal domain (NTD) of the GluN1 promotes heterodimerization between the NTDs of GluN1 and GluN2, whereas the $109 \mathrm{C}$ mutation in the adjacent residue stabilizes the homodimer of the NTD of GluN1. The crystal structure of the NTD of GluN1 revealed the mechanism underlying the biochemical properties of these mutants. Effects of these mutations on the maturation of heteromeric NMDA-Rs were investigated using a receptor trafficking assay. Our results suggest that the NTDs of the GluN1 subunit initially form homodimers and the subsequent dimer dissociation is critical for forming heterotetrameric NMDA-Rs containing GluN2 subunits, defining a molecular determinant for receptor assembly. The domain arrangement of the dimeric NTD of GluN1 is unique among the ionotropic glutamate receptors and predicts that the structure and mechanism around the NTDs of NMDA-Rs are different from those of the homologous AMPA and kainate receptors.
\end{abstract}

\section{Introduction}

NMDA receptors (NMDA-Rs) are ligand-gated ion channels of the glutamate receptor family that are critical for normal brain function (Dingledine et al., 1999; Cull-Candy and Leszkiewicz, 2004), and their dysfunction is implicated in various neurological and psychiatric disorders such as mental retardation, epilepsy, limbic encephalitis, schizophrenia, and ischemic brain damage (Kemp and McKernan, 2002).

Seven genes (GluN1, GluN2A-D, and GluN3A, B) encode the subunits of NMDA-Rs (Moriyoshi et al., 1991; Monyer et al., 1992; Andersson et al., 2001). Mature NMDA-Rs are heteromers that require the inclusion of the obligate subunit GluN1 to form functional channels (Monyer et al., 1992; Forrest et al., 1994). The subunits of NMDA-Rs share homology and consist of four domains

\footnotetext{
Received Nov. 18, 2010; revised Jan. 5, 2011; accepted Jan. 11, 2011.

This study was supported by the National Alliance for Research on Schizophrenia and Depression (NARSAD) Young Investigator Award (to T.N.) and National Institutes of Health (NIH) Grant R01HD061543 (to T.N.). We thank Shigetada Nakanishi and Yasunori Hayashi for the glutamate receptor cDNA clones. The University of California San Diego (UCSD) Cryo-Electron Microscopy Facility was supported by NIH Grants 1S10RR20016 and GM033050 (to T. S. Baker, UCSD) and by the Agouron Institute (to UCSD). A.N.F. was supported by NIH Training Grant T32GM007240-34. K.Y.B. thanks H.A. and Mary K. Chapman Charitable Trust and The Mary K. Chapman Foundation. The X-ray source was supported by NIH Blueprint Grant NS057096. The coordinates and structure factor of the GluN1-NTD was deposited in the Protein Data Bank under the identification code 3041.

*A.N.F. and K.Y.B. contributed equally to this work.

Correspondence should be addressed to either of the following: Terunaga Nakagawa, Department of Chemistry and Biochemistry, University of California San Diego, 9500 Gilman Drive, La Jolla, CA 92093, E-mail: nakagawa@ucsd.edu; or Senyon Choe, Structural Biology Laboratory, 10010 North Torrey Pines Road, Salk Institute, La Jolla, CA 92037, E-mail: choe@salk.edu.

DOI:10.1523/JNEUROSCI.6041-10.2011

Copyright $\odot 2011$ the authors $\quad 0270-6474 / 11 / 313565-15 \$ 15.00 / 0$
}

(see Fig. 1A). The N-terminal domain (NTD) and the ligandbinding domain (LBD) form the extracellular domain. The channel pore-forming transmembrane domain (TMD) consists of three membrane spanning segments (M1, M3, and M4) and one re-entrant loop (M2) (Hollmann et al., 1994). A cytoplasmic C-terminal domain (CTD) interacts with proteins that regulate receptor signaling, anchoring, and trafficking (Scannevin and Huganir, 2000).

The crystal structure of the homologous AMPA receptor (AMPA-R) (Sobolevsky et al., 2009) suggests that the mature NMDA-Rs are tetramers in which the subunits are arranged as a dimer of dimers. In NMDA-R, the LBDs form a pair of heterodimers made of GluN1 and GluN2 subunits (Furukawa et al., 2005). The precise architecture of the NTDs of the mature NMDA-R is unknown. However, the monomeric crystal structure of the GluN2B NTD (Karakas et al., 2009) raises the possibility that the structure and the mechanism around the NTDs are different between AMPA-R and NMDA-Rs (Stroebel et al., 2011).

The NTDs of NMDA-Rs modulate channel gating by binding to $\mathrm{Zn}^{2+}$ ions and ifenprodil (Choi and Lipton, 1999; Low et al., 2000; Paoletti et al., 2000; Perin-Dureau et al., 2002; Gielen et al., 2008). The NTDs also play critical roles in the subunit assembly (Kuusinen et al., 1999; Leuschner and Hoch, 1999; Ayalon and Stern-Bach, 2001; Meddows et al., 2001; Greger et al., 2007; Shanks et al., 2010). The subunit composition of NMDA-Rs is developmentally regulated, defines receptor architecture, and dictates the gating characteristics and synaptic plasticity (CullCandy and Leszkiewicz, 2004). However, the precise mechanism of the subunit assembly of heterotetrameric NMDA-R is unclear and controversial (Traynelis et al., 2010). 
It has been suggested that the GluN1 forms homodimers during the assembly of heterotetrameric NMDA-Rs (Qiu et al., 2005; Atlason et al., 2007), while other studies favor a model in which the NTDs of the GluN1 and GluN2 form heterodimers in the mature receptors (Schüler et al., 2008; Gielen et al., 2009; Sobolevsky et al., 2009). To provide a consistent explanation to these differing models, we introduced point mutations into the NTD of GluN1 and investigated the consequence of changing the state of the NTDs on the assembly of NMDA-Rs. The phenotypes and the underlying mechanisms of these mutants were studied by combining cell biology, biochemistry, single particle electron microscopy (EM), and x-ray crystallography. Our results revealed that the initial dimerization of the GluN1-NTD and the subsequent separation of the dimer are critical for heterotetramerization of the NMDA-R.

\section{Materials and Methods}

The recombinant DNA constructs. Rat cDNA clones of GluN1, GluN2A, GluN2B, and GluA1 were used. The NTD fragments used were (in amino acids); GluN1 (1-393), GluN2A (1-404), GluN2B (1-401), and GluA1 (1-390). Y109C and T110A mutations were generated by QuikChange in vitro mutagenesis protocol (Stratagene). The splice variant of the GluN1NTD used in this study does not contain the reported insertion (Sugihara et al., 1992). Various fragments were subcloned into modified pIRESEGFP, modified pIRES-mCherry, and pTREtight (Clontech) vectors (where IRES is internal ribosome entry site, EGFP is enhanced green fluorescent protein, and TRE is Tet-responsive element). The epitope tags [hemagglutinin (HA), FLAG, and $8 \mathrm{His}$ ] were placed at the C-terminal end of the NTD constructs. In the cell surface expression assays, the full-length GluN2 subunits were tagged with FLAG epitope between the signal peptide cleavage site and the NTD. To coexpress more than two full-length NMDA-R subunits, doxycycline (DOX)-inducible dual expression plasmids were generated. The sequences of all the DNA fragments generated by PCR were verified.

The pIRES-EGFP vector (Clontech) was modified to contain a polyglycine linker, thrombin site, and an HA tag followed by a stop codon $\left(^{*}\right)$ between the SalI and BamHI sites (VDGGGGGLVPRGSYPYDVPDYASS ${ }^{*}$ ). The pIRES-mCherry vector (Clontech) was modified to contain a polyglycine linker, thrombin cut site, and either a FLAG or 8His tag followed by a stop codon between the SalI and BamHI sites (VDGGGGGLVPRGSDYDDDDKSS ${ }^{*}$ or VDGGGGGLVPRGSHHHHHHHHSS ${ }^{*}$ ). The polyglycine linker was introduced to facilitate the thrombin digest. These modifications were done using PCR and synthetic oligonucleotides.

The cDNA fragment GluN1-NTD was amplified by PCR and cloned into the modified pIRES-EGFP or pIRES-mCherry vectors, producing pGluN1NTD-HA-IRES-EGFP, pGluN1-NTD-FLAG-IRES-mCherry, and pGluN1-NTD-8His-IRES-mCherry. Similarly, GluA1-NTD was cloned into the modified pIRES-EGFP vector, producing pGluA1-NTD-FLAGIRES-EGFP. The GluN2B-NTD and GluN2A-NTD cDNA sequences were cloned into the modified pIRES-mCherry vector to create pGluN2B-NTDFLAG-IRES-mCherry, pGluN2B-NTD-8His-IRES-mCherry, or pGluN2ANTD-FLAG-IRES-mCherry. The tagged NTD fragments were subcloned into pTREtight vector to create plasmids for DOXinducible protein expression.

Plasmids that DOX-dependently express full-length GluN1 together with full-length GluN2B or GluN2A constructs were made based on two modified pTRE-tight vectors (Clontech), which we denote as pTRE-A and -B. In pTRE-A, 5'-PacI and $3^{\prime}$-AscI sites were introduced after the XhoI site at base 2 in the pTRE-tight vector using DNA fragments generated by PCR. In pTRE-B, an AscI site was introduced after the XhoI site at base 2, and a PacI site was introduced before the XhoI site at base 602 on the pTRE-tight vector, respectively. The full-length untagged GluN1 construct was cloned into the NotI site of pTRE-A. The full-length rat cDNA of GluN2B or GluN2A tagged with a $3 \times$ FLAG tag (Sigma) at its extreme $\mathrm{C}$ terminus was cloned into pTRE-B between restriction sites EcoRI and EcoRV. The GluN2B-3×FLAG or GluN2A-3×FLAG expression cassette [the Tet-responsive promoter and response element, GluN2
cDNA sequence, and SV40 poly(A) sequence] was excised from the pTRE-B vector and subcloned into the pTRE-A vector containing the GluN1 gene using AscI and PacI. Additionally, to prevent possible promoter interference, a small spacer sequence $(\sim 200 \mathrm{bp})$ was inserted into the AscI site between GluN1 and GluN2 expression cassettes. The dual expression vector ensures that both GluN1 and GluN2 subunits will be expressed in the transfected cells. The coexpression of both proteins by each plasmid was confirmed by Western blotting and immunocytochemistry.

The Y109C and T110A mutations were also introduced into the fulllength GluN1 and were subcloned into the NotI site of pTRE-A, as described above. The dual expressing plasmids that coexpress GluN1 mutant and wild-type (wt) GluN2A/B was created using similar methods as those described above.

$\Delta$ NTD-GluN1 cDNA was created with standard PCR technique by deleting the sequence of NTD after the signal peptide coding region (amino acids 22M-393Q) from GluN1. To create DOX-inducible expression vectors, the above-described GluN1 (wt or $\Delta$ NTD) or GluN2A fragments were inserted into pTRE-A or pTRE-B vectors, respectively. To create dual expressing vectors, the expression cassette from pTRE-AGluN1 (wt or $\Delta$ NTD) and pTRE-B-GluN2A were cut and ligated together using PacI and AscI sites as described above.

Cell culture. The HEK-TetON cells (Clontech) and GnTI(-) HEK cells were used in this study (where HEK is human embryonic kidney and Tet is tetracycline). The HEK-TetON cells, used for DOX-inducible protein expression, stably express reverse Tet transcriptional activator but otherwise phenocopy HEK cells.

Generation of stable cell lines and protein expression. The NTDexpressing plasmids in modified pIRES vectors were transfected into GnTI(-) HEK cell lines using calcium phosphate transfection methods (Chen and Okayama, 1987). For stable cell line generation, we cotransfected each construct with a plasmid that express hygromycin-resistant gene and selected cells with $120 \mu \mathrm{g} / \mathrm{ml}$ hygromycin (Invitrogen) over 2 weeks. Isolated colonies were expanded and screened for expression by monitoring fluorescence. The expression of each NTD was confirmed by Western blotting. Stable cell lines were maintained in high glucose DMEM containing $10 \%$ fetal calf serum (FCS), $10 \mu \mathrm{g} / \mathrm{ml}$ penicillin and streptomycin (Pen/Strep), and $120 \mu \mathrm{g} / \mathrm{ml}$ hygromycin. For protein expression, cells were grown in monolayers. Once the plates were $90-95 \%$ confluent, the medium was collected and replaced with Opti-MEM (Invitrogen) containing $10 \mu \mathrm{g} / \mathrm{ml}$ Pen/Strep. Every $48 \mathrm{~h}$ the medium was removed and centrifuged at $3500 \mathrm{rpm}$ in a JLA-16.25 rotor (Beckman) for $10 \mathrm{~min}$. to remove cell debris. The resulting supernatant was used for purification.

To create a stable GnTI( - ) HEK cell line that express both the GluN1NTD and GluN2B-NTD, we first created a GluN1-NTD-HA-expressing cell line with hygromycin resistance. Next, GluN2B-NTD-FLAG and pCMV Zeocin (Invitrogen) were cotransfected and selected with 250 $\mu \mathrm{g} / \mathrm{ml}$ Zeocin (Invitrogen) over 2 weeks. Isolated colonies were amplified and screened for both GFP and mCherry fluorescence. Protein expression was confirmed by Western blot against the FLAG and HA tags of the GluN2B-NTD and GluN1-NTD, respectively. The dual expressing stable cell lines were maintained in DMEM containing 10\% FCS, 10 $\mu \mathrm{g} / \mathrm{ml}$ Pen/Strep, $120 \mu \mathrm{g} / \mathrm{ml}$ hygromycin, and $150 \mu \mathrm{g} / \mathrm{ml}$ Zeocin.

Protein purification and crystallization of the GluN1-NTD. One liter of culture supernatant of the GluN1-NTD-8His-expressing stable GnTI(-) HEK cell line was adjusted to 1.5 liters by adding $200 \mathrm{~mm}$ sodium phosphate buffer and $1 \mathrm{~m}$ imidazole, $\mathrm{pH}$ 7.5. The final concentrations of sodium phosphate buffer and imidazole were 50 and $25 \mathrm{~mm}$, respectively. The medium was then gravity loaded onto a chelating Sepharose column charged with $\mathrm{Ni}^{2+}$ that was pre-equilibrated with Opti-MEM containing $50 \mathrm{~mm}$ sodium phosphate buffer, $\mathrm{pH} 7.5$, and $25 \mathrm{~mm}$ imidazole. After washing with 10 column volumes of wash buffer ( $50 \mathrm{~mm}$ sodium phosphate buffer, $\mathrm{pH}$ 7.5, $30 \mathrm{~mm}$ imidazole, and $150 \mathrm{~mm} \mathrm{NaCl}$ ), the bound protein was eluted from the column with $20 \mathrm{~mm}$ Tris- $\mathrm{HCl}, \mathrm{pH}$ 7.5, 250 $\mathrm{mm}$ imidazole, and $150 \mathrm{~mm} \mathrm{NaCl}$. The eluted fraction containing GluN1NTD was then adjusted to $20 \mathrm{~mm}$ Tris- $\mathrm{HCl}, \mathrm{pH} 7.5,100 \mathrm{~mm}$ imidazole, $150 \mathrm{~mm} \mathrm{NaCl}$, and $2.5 \mathrm{~mm} \mathrm{CaCl}_{2}$ and digested with thrombin (GE Healthcare) overnight at room temperature to remove the $8 \mathrm{His}$ tag. The protein mixture was dialyzed against $20 \mathrm{~mm}$ Tris- $\mathrm{HCl}, \mathrm{pH}$ 7.5, and 150 
$\mathrm{mm} \mathrm{NaCl}$ and then treated with endoglycosidase $\mathrm{H}$ (Endo $\mathrm{H} ; 1 \mathrm{U}$ of enzyme for $1 \mu \mathrm{g}$ of purified protein; New England BioLabs) overnight at room temperature. The GluN1-NTD was further purified by gel filtration chromatography and concentrated to $10 \mathrm{mg} / \mathrm{ml}$. The concentrated GluN1-NTD was crystallized by the hanging drop vapor diffusion method by mixing $1: 1$ with $4 \mathrm{M}$ formate and $20 \mathrm{~mm}$ tris(2-carboxyethyl)phosphine (TCEP) at $4^{\circ} \mathrm{C}$. To generate crystals for single isomorphous replacement with anomalous scattering (SIRAS) experiments, the GluN1-NTD was cocrystallized with iodine by mixing the concentrated protein $1: 1$ with $4 \mathrm{M}$ formate and $200 \mathrm{~mm}$ sodium iodine at $4^{\circ} \mathrm{C}$.

Data collection and structural refinement. For x-ray diffraction experiments, crystals were taken directly from the droplets with a fiber loop and flash frozen in liquid $\mathrm{N}_{2}$ with a cryoprotectant ( $5 \mathrm{M}$ sodium formate and $10 \%$ glycerol for native crystals and $5 \mathrm{~m}$ sodium formate, $10 \%$ glycerol, and $100 \mathrm{~mm}$ sodium iodine for iodine derivative crystals). The diffraction data of the Endo $\mathrm{H}$-treated proteins containing minimal glycosylation were collected for iodine and native crystals at the Salk Institute's Structural Biology Laboratory on a Rigaku MicroMax-007 at a resolution of 3.4 A. The iodine and native $\mathrm{x}$-ray datasets comprise 330,111 and 192,346 reflections, respectively, and were integrated and scaled using the program HKL-2000, version 0.98.692i (Otwinowski and Minor, 1997). The space group of GluN1-NTD was found to be $P 3_{1} 21$ with the following unit cell dimensions: $a, b=164.7, c=147.3, \alpha, \beta=90, \gamma=120$.

The crystal structure of GluN1-NTD was solved by a combination of SIRAS and single wavelength anomalous dispersion using the iodine signal. Phase calculation was completed using the autoSHARP (de La Fortelle and Bricogne, 1997; Vonrhein et al., 2007) software in the CCP4 program suite 6.1.13 (Collaborative Computational Project, Number 4, 1994). The iodine atom sites were located with the program SHELXD (Schneider and Sheldrick, 2002), and solvent flattening was performed in SOLOMON (Abrahams and Leslie, 1996), yielding electron density maps that permitted the manual building of the protein chains using the program COOT, version 0.6.2-pre-1 (Emsley et al., 2010). The final solution contains three molecules in the asymmetric unit, each containing five Asn-linked $N$-acetylglucosamine molecules. Using the native dataset, refinement of the structure was completed after multiple cycles of refinement using REFMAC, version 5.5.0109 (Murshudov et al., 1997) with various weighting terms and noncrystallographic symmetry restraints and converged to $R$ and $R_{\text {free }}$ of 0.23 and 0.28 , respectively.

The final data processing and refinement statistics are listed in Table 1. The root mean squared deviations in bond lengths and bond angles are $0.008 \AA$ and $1.269^{\circ}$, respectively. The Ramachandran plot calculated for the final model using the PROCHECK software (Laskowski et al., 1993) illustrates that the final conformations for $82.4 \%$ of the residues are located in the most favored region, and $14.7 \%$ of the residues are located in the additionally allowed regions. All water molecules have a density of $1 \sigma$ or greater in the $2 F_{\mathrm{o}}-F_{\mathrm{c}}$ map. All structure figures were generated using PyMOL (version 1.2r3; Schrödinger). The space fill of the binding pockets "I" and "Z" were generated using the program "Hollow" (Ho and Gruswitz, 2008).

Analytical gel filtration chromatography. FLAG or nickel affinitypurified NTD proteins were loaded onto a Superdex $200 \mathrm{HR}$ column (GE Healthcare). The column was calibrated by the following protein standards: ribonuclease A, aldolase, ferritin, and albumin. The Stokes radius (Sr) or the molecular weight (MW) was determined by plotting the $K_{\mathrm{av}}$ versus the $\log (\mathrm{Sr})$ or $\log (\mathrm{MW})$. The $K_{\mathrm{av}}$ of each protein was determined by the equation $K_{\mathrm{av}}=\left(V_{\mathrm{e}}-V_{0}\right) /\left(V_{\mathrm{t}}-V_{0}\right)$, where $V_{\mathrm{e}}$ is elution volume, $V_{0}$ is void volume, and $V_{\mathrm{t}}$ is total volume. The peak elution volumes of the 8His-tagged and FLAG-tagged NTDs were indistinguishable.

Negative stain electron microscopy. The NTDs were expressed as secreted entities in HEK GnTI(-) cells. Because GluN2B-NTD does not secrete efficiently on its own, it was obtained from a stable HEK GnTI(-) cell line that expresses both the GluN1-NTD and GluN2B-NTD. The GluN2B-NTD was purified to homogeneity. Specifically, the copurified GluN1-NTD is washed away during the column-washing step in the affinity chromatography. This is possible because the affinity between GluN1-NTD and GluN2B-NTD is not high enough to survive extensive wash. The purified GluA1-NTD, GluN1-NTD (Y109C or wild type), and GluN2B-NTD were negatively stained with $0.7 \%$ uranyl formate as de-

Table 1. Data collection and model refinement statistics

\begin{tabular}{lll}
\hline & GluN1-NTD (iodine) & GluN1-NTD (native) \\
\hline Space group & $P 3_{1} 21$ & $P 3_{1} 21$ \\
Unit cell parameters $\left(\AA^{\circ}{ }^{\circ}\right)$ & $a, b=164.7$ & $a, b=164.7$ \\
& $c=147.0$ & $c=147.3$ \\
& $\alpha, \beta=90.0$ & $\alpha, \beta=90.0$ \\
& $\gamma=120.0$ & $\gamma=120.0$
\end{tabular}

\section{Data collection statistics Beamline}

Wavelength $(\AA)$

Resolution range $(\AA)$

Observed reflections

Unique reflections

Redundancy

$R_{\text {merge }}(\%)$

Mean $/ / \sigma(l)$

Completeness (\%)

\section{Refinement statistics}

$R_{\text {cryst }}(\%)$

$R_{\text {free }}(\%)^{a}$

Average $B$ factor $\left(\AA^{2}\right)$ Bond lengths $(\AA)$

Bond angles $\left({ }^{\circ}\right)$

Number of atoms total

Protein

Water

Chlorine

lodine

$\mathrm{N}$-Acetylglucosamine

Ramachandran plot (\%):

Most favored

Additional allowed

Generously allowed

Disallowed allowed
Root mean square deviation

Rigaku MicroMax-007
(Salk Institute, SBL-C)
1.5418
$100-3.40(3.46-3.40)$
330111
32227
$10.2(10.1)^{b}$
$17.3(68.5)^{b}$
$15.0(3.4)^{b}$
$99.9(100.0)^{b}$

Rigaku MicroMax-007

(Salk Institute, SBL-C) 1.5418

$100-3.40(3.46-3.40)$

192346

32023

$6.0(5.8)^{b}$

$14.6(63.1)^{b}$

$12.9(2.8)^{b}$

$99.9(100.0)^{b}$

\begin{tabular}{cc}
21.7 & 22.9 \\
28.3 & 27.9 \\
76.284 & 73.06 \\
& \\
0.010 & 0.008 \\
1.484 & 1.269 \\
8983 & 8968 \\
8725 & 8725 \\
22 & 25 \\
7 & 8 \\
19 & 0 \\
210 & 210 \\
& \\
79.8 & 82.4 \\
15.2 & 14.7 \\
2.4 & 1.7 \\
2.7 & 1.1 \\
\hline
\end{tabular}

${ }^{a}$ Calculated from $5 \%$ of data not used in refinement.

${ }^{b}$ Numbers in parentheses correspond to highest resolution shell $(\AA)$.

scribed by Ohi et al. (2004). Images were recorded using a FEI Sphera electron microscope operated at an acceleration voltage of $200 \mathrm{keV}$ at a magnification of $\times 50,000$ and defocus values ranging form -1.5 to -1.8 $\mu \mathrm{m}$. All images were recorded using SO-163 film and developed with a Kodak D-19 developer at full strength for $12 \mathrm{~min}$ at $20^{\circ} \mathrm{C}$. The electron micrographs were digitized with a CoolScan 9000 (Nikon) using a step size $6.35 \mu \mathrm{m}$, and the pixels were binned by a factor of 3. As a result, the specimen level pixel size was at $3.8 \AA$. To generate projection averages, particles were interactively selected using the WEB display program in SPIDER (Frank et al., 1996). Using SPIDER, projection averages were calculated from windowed small images of $64 \times 64$ pixels over 8 cycles of $k$-means classification and multiference alignment specifying 100 classes. In the case where the total pixels occupied by the particles in the class averages were calculated, each class average was converted to binary mode in ImageJ, and the total pixels were calculated using the area measure function.

Cross-linking experiments of NTDs. Wild-type, Y109C, and N70C GluN1-NTD in modified pIRES-EGFP vectors containing C-terminal HA tags were individually transfected transiently into GnTI(-)HEK cells by a calcium phosphate method (Chen and Okayama, 1987). After $24 \mathrm{~h}$ posttransfection the medium was changed to Opti-MEM containing 10 $\mu \mathrm{g} / \mathrm{ml}$ Pen/Strep. After $48 \mathrm{~h}$ the medium was collected and centrifuged at $3500 \mathrm{rpm}$ for $10 \mathrm{~min}$ to remove cell debris. The preclarified medium was then added to either nonreducing SDS-PAGE sample buffer (final concentrations of $10 \%$ glycerol, $50 \mathrm{~mm}$ Tris, $\mathrm{pH} 7.6,8 \%$ SDS) or reducing SDS-PAGE sample buffer [final concentrations of $10 \%$ glycerol, $50 \mathrm{~mm}$ Tris, $\mathrm{pH}$ 7.6, 8\% SDS, and $100 \mathrm{~mm}$ dithiothreitol (DTT)]. Medium samples were then boiled for $5 \mathrm{~min}$ at $100^{\circ} \mathrm{C}$ before subjection to SDS-PAGE. The total cell lysate for each GluN1-NTD sample was collected at the 
same time using the following procedure: the remaining cells attached to the cell culture plates of each sample were resuspended in PBS, pH 7.4, directly lysed in reducing SDS-PAGE sample buffer, and boiled for $5 \mathrm{~min}$. at $100^{\circ} \mathrm{C}$.

In the experiment in Figure $8 C$ where the GluN2B-NTD expression was varied in the presence of the GluN1-NTD-Y109C, increasing amounts of pGluN2B-NTD-FLAG-IRES-mCherry plasmids $(3,8,15$, or $23 \mu \mathrm{g})$ were mixed with a constant amount $(2 \mu \mathrm{g})$ of pGluN1NTD(Y109C)-HA-IRES-EGFP. In each transfection empty vector pIRES-mCherry was supplemented to keep the total amount of DNA at $25 \mu \mathrm{g}$. The remaining procedures were done as described above.

Cross-linking experiments of full-length NMDA-Rs. HEK-TetON cells (Clontech) were plated on gelatin-coated 6-well plates at a density of $1 \times$ $10^{6}$ cells/well. The following day, plasmids that express single or dual full-length NMDA-R subunits were transfected into the cells using a calcium phosphate transfection method (Chen and Okayama, 1987). After transfection, cells were treated with $7.5 \mu \mathrm{g} / \mathrm{ml}$ DOX and $1 \mathrm{~mm}$ sodium butyrate to induce NMDA-R expression. The induced cells were maintained in $1 \mathrm{~mm}$ kynurenic acids (Tocris Bioscience), $10 \mathrm{~mm} \mathrm{MgSO}_{4}$, and $10 \mu \mathrm{M}(+) \mathrm{MK} 801$ (Ascent Scientific) to prevent NMDA-Rmediated cell death. Detergent extraction of NMDA-R from cells was done following the specifications of Sobolevsky et al. (2009). Specifically, cells were harvested $24 \mathrm{~h}$ after transfection and solubilized by nutating at $4^{\circ} \mathrm{C}$ for $1 \mathrm{~h}$ in $20 \mathrm{~mm}$ HEPES, pH 7.2, $150 \mathrm{~mm} \mathrm{NaCl}, 2 \%$ dodecyl maltoside (DDM; Anatrace) supplemented with protease inhibitors $(1 \mathrm{~mm}$ PMSF and $10 \mu \mathrm{g} / \mathrm{ml}$ leupeptin, atropinin, benzamidine, and pepstatin A). Cell lysates were clarified by ultracentrifugation at 40,000 $\mathrm{rpm}$ for 40 min at $4^{\circ} \mathrm{C}$. Supernatants were mixed with SDS sample buffer with or without $100 \mathrm{~mm}$ DTT.

To coimmunoprecipitate GluN2B with full-length wild-type, N70C, or Y109C GluN1, cell lysates $(1 \mathrm{ml})$ were incubated overnight with $50 \mu \mathrm{l}$ of M2-FLAG-conjugated Sepharose resin pre-equilibrated with $20 \mathrm{~mm}$ HEPES, pH 7.2, $150 \mathrm{~mm} \mathrm{NaCl}$, and 2\% DDM supplemented with protease inhibitors ( $1 \mathrm{~mm}$ PMSF and $10 \mu \mathrm{g} / \mathrm{ml}$ leupeptin, atropinin, benzamidine, and pepstatin A). The resin was gently spun down and washed twice with $400 \mu$ l of $20 \mathrm{~mm}$ HEPES, pH 7.2, $150 \mathrm{~mm} \mathrm{NaCl}$, and 2\% DDM. The protein was eluted by incubating the resin in $20 \mathrm{~mm}$ HEPES, pH 7.2, $150 \mathrm{~mm} \mathrm{NaCl}, 2 \% \mathrm{DDM}$, and $0.25 \mu \mathrm{g} / \mathrm{ml} 3 \times$ FLAG peptide (Sigma). Elution fractions were mixed with SDS sample buffer with or without 100 mM DTT.

In the experiment in Figure 8D, a constant amount of GluN1-Y109Cexpressing plasmid $(10 \mu \mathrm{g})$ was mixed with increasing amounts of GluN2B-expressing plasmids $(0,1,5$, or $15 \mu \mathrm{g})$. The empty vector pTREtight (Clontech) was supplemented to keep the total amount of DNA per transfection at $25 \mu \mathrm{g}$. The remaining procedures were done as described above.

Coimmunoprecipitation of the GluN1-NTD with GluN2-NTDs. GnTI(-) HEK cells were transiently transfected with equal amounts of DNA of pGluN1-NTD-HA-IRES-EGFP and pGluN2A-NTD-FLAG-IRESmCherry or pGluN2B-NTD-FLAG-IRES-mCherry by standard calcium phosphate methods. After $24 \mathrm{~h}$, the medium was changed to Opti-MEM (Invitrogen) containing $10 \mu \mathrm{g} / \mathrm{ml} \mathrm{Pen/Strep.} \mathrm{The} \mathrm{cells} \mathrm{were} \mathrm{then} \mathrm{main-}$ tained in Opti-MEM for $48 \mathrm{~h}$, at which point the medium was clarified by centrifugation at $3500 \mathrm{rpm}$ for $10 \mathrm{~min}$ to remove cell debris. Preequilibrated $50 \mu \mathrm{l}$ of M2-FLAG resin was added to the medium (10 ml) and allowed to bind overnight at $4^{\circ} \mathrm{C}$. The samples were spun at $500 \mathrm{rpm}$. The supernatant was then removed but saved, and the resin was collected and washed twice with $400 \mu \mathrm{l}$ of wash buffer (20 mM HEPES, pH 7.4, 150 $\mathrm{mm} \mathrm{NaCl}$ ). The protein was then eluted by incubating the resin with the wash buffer containing $0.25 \mu \mathrm{g} / \mathrm{ml}$ FLAG peptide (Sigma).

Cell surface staining and quantification. HEK-TetON cells on poly-Llysine were transfected with pTREtight expression vectors encoding $\triangle$ NTD-GluN1 and FLAG-tagged GluN2A in combination with empty pTREtight vector (mock) or with pTREt-GluN1-NTD-HA(wt/Y109C/ T110A) using a standard calcium phosphate method. The FLAG tag in GluN2 subunits is located after the signal peptide cleavage site and before the NTD. Twenty-four hours after induction with $7.5 \mu \mathrm{g} / \mathrm{ml}$ DOX and 1 mM Na-butyrate, cells were live labeled with FLAG M2 antibody (Sigma) in DMEM (Mediatech) containing $10 \mu \mathrm{M}(+) \mathrm{MK} 801$ (Ascent) for $10 \mathrm{~min}$ at $37^{\circ} \mathrm{C}$ in a $5 \% \mathrm{CO}_{2}$ incubator. Then cells were washed in DMEM following fixation with $4 \%$ formaldehyde in $0.1 \mathrm{~m}$ phosphate buffer, $\mathrm{pH} 7.4$.

After three PBS washes, the total GluN1 was labeled with anti-GluN1 antibody (against $\mathrm{C} 1$ exon, rabbit polyclonal) diluted in 1XGBD $(0.2 \%$ gelatin, $0.6 \%$ Triton $\mathrm{X}-100,33 \mathrm{~mm}$ phosphate buffer, $\mathrm{pH} 7.4$, and $0.9 \mathrm{M}$ $\mathrm{NaCl}$ ).

Anti-rabbit IgG antibody conjugated with Alexa Fluor 488 and antimouse IgG conjugated with Alexa Fluor 568 were used as the secondary antibodies (Invitrogen). Cells were imaged using a fluorescence microscope (Olympus) with a $10 \times$ objective lens. Images were recorded under the identical condition on a CCD camera (Hamamatsu Orca). Surface labeled fluorescence density was calculated from each cell after subtracting the background signal and used for further statistical analysis. The ImageJ software was used for image processing. Statistical analysis was done using two-tailed Student's $t$ test.

To double stain the GluN1-NTD and GluN2B-NTD in Figure $1 D$, constructs in pIRES-EGFP- or mCherry-based vectors were not used to prevent the overlap of the emission wavelengths employed to detect the NTDs. To overcome this problem, the NTDs were cloned into pTREtight for expression. DOX-inducible HEK-TetOn cells (Clontech) were transiently transfected with a pTREtight plasmid either singly expressing GluN2B-NTD or dually expressing GluN2B-NTD and GluN1-NTD by a standard calcium phosphate method. Cells were induced with $7.5 \mu \mathrm{g} / \mathrm{ml}$ DOX for $24 \mathrm{~h}$, washed with warm PBS, and fixed with $4 \%$ formaldehyde (Polysciences) in $0.1 \mathrm{~m}$ phosphate buffer, $\mathrm{pH}$ 7.4. The cells were then probed with anti-FLAG (monoclonal antibody from Sigma) and anti-HA (polyclonal antibody from Santa Cruz Biotechnology) antibodies. Alexa Fluor 488-conjugated anti-mouse IgG and Alexa Fluor 568 anti-rabbit IgG were used as secondary antibodies (Invitrogen). Imaging was performed with a CCD camera (Hamamatsu Orca) using an Olympus fluorescence microscope $(40 \times$ objective lens).

\section{Results}

\section{The GluN1-NTD rescues the trafficking defect caused} by $\Delta$ NTD-GluN1

The GluN2 requires GluN1 to traffic to the cell surface (McIlhinney et al., 1998). Consistently, GluN2 reached the cell surface when it was coexpressed with the wild-type GluN1 but not with the GluN1 that lacks the NTD ( $\Delta$ NTD-GluN1) (Meddows et al., 2001) (Fig. 1B). To further characterize the function of the NTD in receptor assembly, we tested whether the isolated GluN1-NTD can rescue the impaired cell surface expression of GluN2 caused by the coexpressed $\Delta$ NTD-GluN1. Interestingly, when the soluble GluN1-NTD was coexpressed in HEK-TetON cells with wildtype GluN2A and $\triangle$ NTD-GluN1, the surface expression of GluN2A was rescued (Fig. $1 B, C$ ). The GluN2 surface expression was absent when $\Delta$ NTD-GluN1 was omitted (Fig. $1 B, C$ ). These results suggest that the isolated GluN1-NTD is functional and cooperates with $\Delta$ NTD-GluN1 to promote surface expression of the GluN2A. Furthermore, the results establish a cellular assay to investigate the mechanism of the GluN1-NTD function in NMDA-R assembly.

\section{The GluN1-NTD is required for the secretion of GluN2B-NTD}

The isolated GluN1-NTD secretes as a soluble entity when expressed in GnTI(-) HEK cells (Fig. 1E), a mutant HEK cell line lacking an enzyme that mediates complex mannose glycosylation (Reeves et al., 2002). In contrast, the GluN2B-NTD was incapable of secreting on its own but required the GluN1-NTD for secretion (compare Fig. 1, compare E, F). A similar phenomenon was reported for the GluN2A-NTD (Qiu et al., 2009). The GluN1NTD and GluN2B-NTD proteins partially colocalized when coexpressed (Fig. 1D), and furthermore the two proteins coimmunoprecipitated (Fig. $1 G$ ). These results establish direct interaction between the NTDs of GluN1 and GluN2B. Similar 


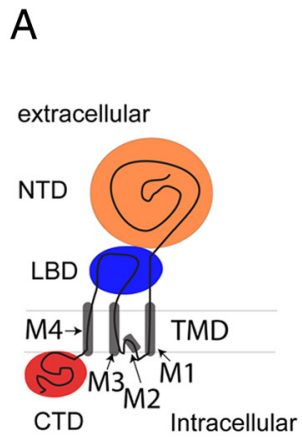

C

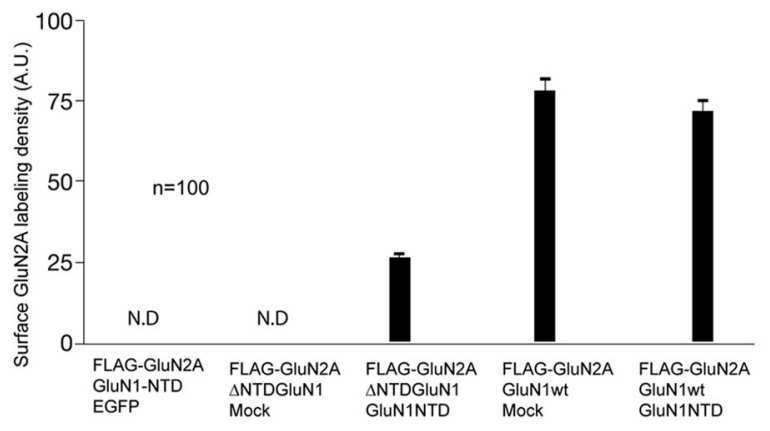

$E$

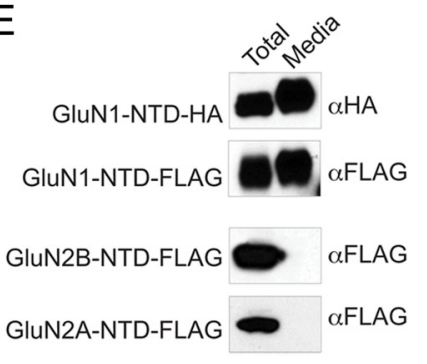

B
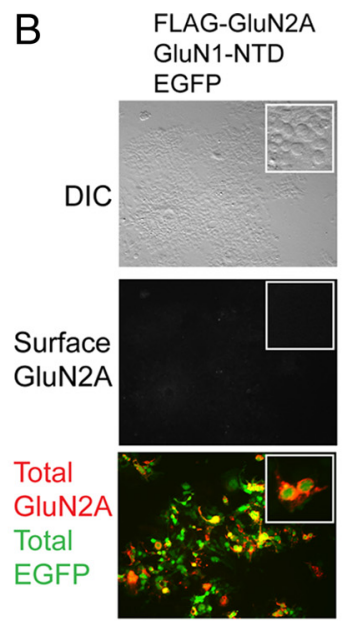

$\mathrm{F}$

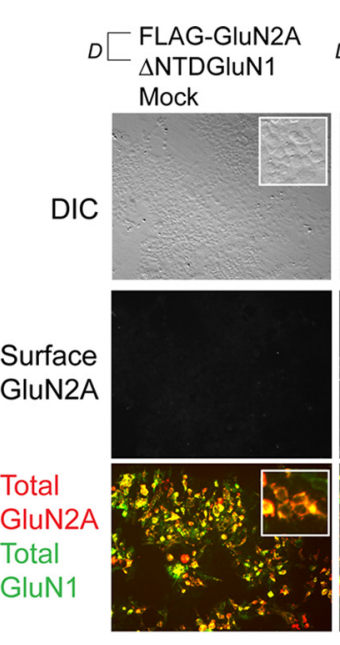

D

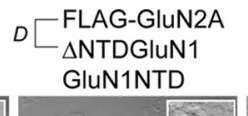

$D \leftarrow$ FLAG-GluN2A
Mock
Mock
Mok
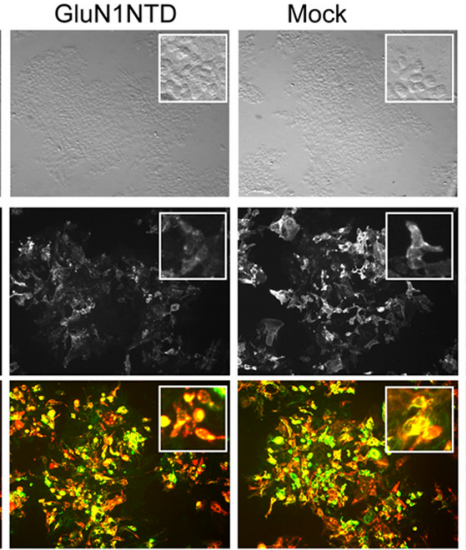

GluN2B-NTD

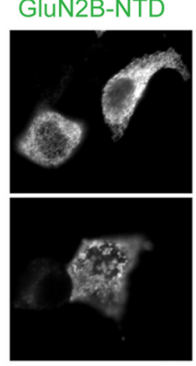

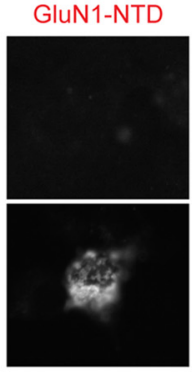

-FLAG-GluN2A

-GluN1wt GluN1NTD

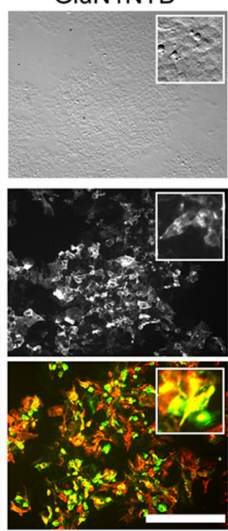

Merge

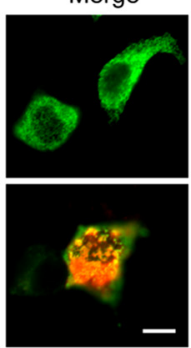

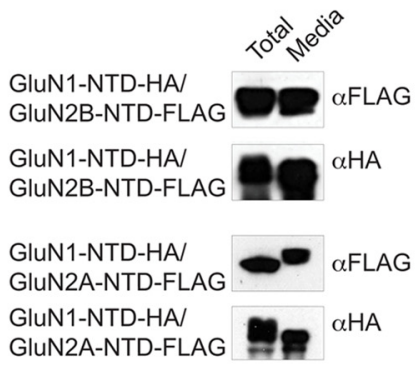

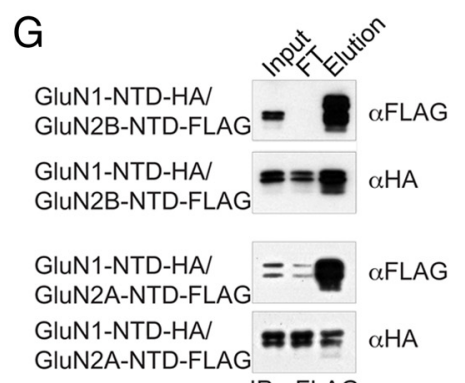

IP: $\alpha \mathrm{FLAG}$

Figure 1. The NTD of the GluN1 interacts with the NTDs of GluN2. A, Domain organization of the full-length NMDA-R. The N-terminal domain, ligand-binding domain, transmembrane domain (indicating M1, M2, M3, and M4 transmembrane helices), and C-terminal domain are indicated. $\boldsymbol{B}$, Images of HEK-TetON cells cotransfected with pTREtight-derived plasmids that express the proteins indicated at the top of each column. " $D$ " indicates that the two proteins were expressed using a dual expressing plasmid. The empty plasmid that does not express any protein is indicated as "Mock." EGFP-expressing plasmid was used as a mock plasmid in the left column. Top row, Differential interference contrast (DIC) image. Middle row, Surface staining of the identical field as the DIC image. Anti-FLAG antibody was used to detect FLAG-GluN2A. Bottom row, The merged total staining demonstrating efficient coexpression, done as separate experiment from the surface staining. Insets, Representative magnified images. Scale bar, $200 \mu \mathrm{m}$. C, Quantification of surface-labeled GluN2A from B. N.D., Not determined because there was no signal. Sample size, $n=100$ cells. D, Immunofluorescent images of HEK-TetON cells expressing GluN1-NTD-HA alone (top row) or with GluN2B-NTD-FLAG (bottom row). Green, GluN2B; red, GluN1-NTD. pTREtight vector was used to express the NTDs. Scale bar, $10 \mu \mathrm{m}$. E, Western blot of the total cell lysate (Total) and the culture supernatant (Media) of GnTI(-) HEK cells that express the NTDs of GluN1 and GluN2 subunits, as indicated at the left of each row. The antibodies used to probe are indicated ( $\alpha \mathrm{HA}$ and $\alpha \mathrm{FLAG}$ ). $\boldsymbol{F}$, Western blot of the total cell lysate (Total) and the culture supernatant (Media) of GnTI( - ) HEK cells that are cotransfected with plasmids that express the NTDs of GluN1 and GluN2 subunits, as indicated at the left of each row. G, Western blot demonstrating the coimmunoprecipitation (Co-IP) of the GluN2A(or 2B)-NTD-FLAG with the GluN1-NTD-HA. Co-IP was done using anti-FLAG antibody (IP: $\alpha$ FLAG). Input, flow through (FT), and elution fractions are shown.

results were obtained when the GluN2A subunit was used instead of GluN2B (Fig. 1 E-G) and when HEK or HEK-TetON cells were used instead of $\mathrm{GnTI}(-)$ HEK cells (data not shown).

\section{The T110A mutation facilitate heterodimer formation}

By comparing the sequence of the GluN1-NTD and the dimeric crystal structures of the homologous NTDs of GluA2 and GluK2 (Jin et al., 2009; Kumar et al., 2009), we searched for a point mutation in the GluN1-NTD that promotes secretion of GluN2B-NTD by enhancing heterodimerization with GluN2BNTD. In detail, the homodimeric GluA2-NTD and GluK2-NTD utilizes helices $\alpha 2$ and $\alpha 3$ for dimerization. We assumed that if
GluN1-NTD and GluN2B-NTD heterodimerize, the corresponding $\alpha$ helices in the GluN1-NTD will be used. We therefore mutated several residues within this region of the GluN1-NTD and identified that, compared to the wild type, a mutation converting threonine 110 to alanine (T110A) promotes the cosecretion of GluN2-NTD in HEK cells. Furthermore, the GluN1-NTD-T110A coimmunoprecipitated with the GluN2-NTD better than wild-type GluN1-NTD (Fig. 2A-C). Consistently, in the gel filtration chromatography we detected a distinct peak of heterodimers formed of GluN1-NTD-T110A and GluN2B-NTD that was undetectable in the absence of the GluN2B-NTD (Fig. 2D, left). The Western blotting clearly demonstrates that both GluN1T110A and GluN2B co- 
purify in the fraction consistent with the heterodimers (Fig. 2D, right). These results establish the GluN1-NTD-T110A as a mutation that promotes heterodimer formation with the GluN2-NTD.

\section{Reduced surface expression of NMDA-Rs in T110A mutants}

Because the GluN1-NTD is required for the GluN2A-NTD to enter the secretory pathway, we predicted that promoting the heterodimer formation by introducing the T110A mutation in GluN1 would accelerate receptor assembly and trafficking. Surprisingly, compared to the wild-type GluN1-NTD, the GluN1-NTD-T110A had significantly lower ability to restore the impaired cell surface expression of GluN2A caused by the $\Delta$ NTD-GluN1 (Fig. $2 E, F$ ). These results indicate that promoting the heterodimer formation of the NTD is insufficient to accelerate the assembly of NMDA-R, suggesting the existence of additional mechanisms.

\section{The single particle EM structures of NTDs of NMDA-Rs}

The NTDs of AMPA-R subunits homodimerize during assembly, and dimeric NTDs are observed in the mature receptors (Nakagawa et al., 2005; Sobolevsky et al., 2009; Shanks et al., 2010). We investigated the oligomerization state of the purified NTDs of NMDA-Rs by negative staining and single particle EM analysis. The majority of the GluN2B-NTD particles (Fig. 3C) were smaller than the particles of the NTD of AMPA-R subunit GluA1 (Fig. 3A, $B, E$ ), which is known to form stable dimers (Jin et al., 2009). Furthermore, the majority of the GluN1-NTD particles were about the same size as the GluN2B-NTD, which is known to be a monomer (Karakas et al., 2009) (Fig. $3 C-E)$. We also noted that $\sim 5 \%$ of the GluN1-NTD particles adopted size and shapes in agreement with a dimer (Fig. $3 D, E$, arrows). We interpret that a small but significant population of dimeric wild-type GluN1-NTD exists in solution. These results indicate that the GluN1-NTD is capable of forming dimers in solution, which is in stark contrast to the GluN2B-NTD that was monomeric (Karakas et al., 2009).

\section{The crystallization of the GluN1-NTD}

To gain insight into the function of the GluN1-NTD and to further investigate the interdomain contacts in the dimeric GluN1-NTD, we determined the $\mathrm{x}$-ray structure of the GluN1NTD that was expressed as a secreted entity in the culturing medium of GnTI(-) HEK cells. The GluN1-NTD crystallized in the space group $P 3_{1} 21$ with three copies of each molecule per asymmetric unit. The final structure was refined and in-
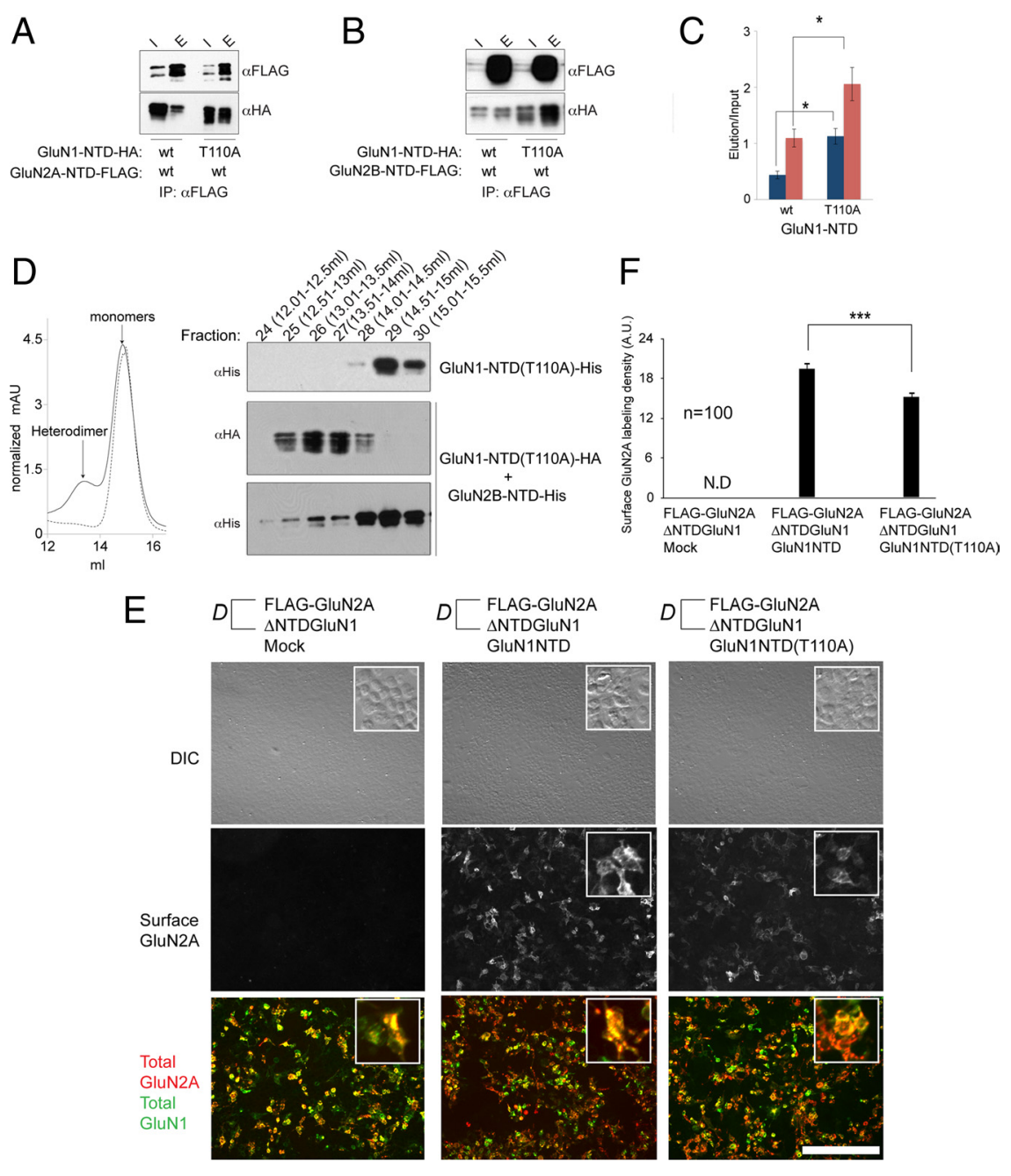

Figure 2. The T110A mutation promotes heterodimerization of the NTDs of GluN1 and GluN2. A, The HA tagged wt or mutant (T110A) GluN1-NTD was coexpressed with GluN2A-NTD-FLAG in GnTI(-) HEK cells. The secreted proteins were immunoprecipitated using the anti-FLAG antibody. Input (I) and elution (E) fractions are shown in the Western blots. The antibodies used to probe are indicated ( $\alpha \mathrm{HA}$ and $\alpha \mathrm{FLAG}$ ). $\boldsymbol{B}$, Experiment similar to that shown in $\boldsymbol{A}$, except that GluN2B-NTD was used instead of GluN2ANTD. $C$, The ratio of the elution versus input in $\boldsymbol{A}$ and $\boldsymbol{B}$ are quantified and summarized in a histogram. Blue, GluN2A-NTD; red, GluN2B-NTD. Asterisk $\left(^{*}\right.$ ) indicates $p<0.05, n=3$. Note the increased association between the NTDs of GluN1 and GluN2 when T110A mutation was introduced. D, GluN2B-NTD-8His and GluN1-NTD (T110A)-HA were coexpressed in GnTI(-) HEK cells and the secreted proteins were purified using the His tag. Representative gel filtration chromatographs of the purified proteins are shown by the solid line. The dotted line is the chromatograph of the purified GluN1-NTD (T110A)-8His. Arrows indicate the elution volumes of the heterodimer and monomers. Western blots of peak fractions are shown to the right (the ranges of elution volumes for each fraction are shown above the fraction number). The antibodies used to probe are indicated ( $\alpha \mathrm{HA}$ and $\alpha \mathrm{His}$ ). E, Images of HEK-TetON cells cotransfected with pTREtight-derived plasmids that express the proteins indicated at the top of each column. " $D$ " indicates that the two proteins were expressed using a dual expressing plasmid. The empty plasmid without an insert was used in the left column as a negative control (Mock). Top row, Differential interference contrast (DIC) image. Second row, Surface staining of the identical field using anti-FLAG antibody that detects the full-length FLAG-GluN2A on the cellular surface. Bottom row, The merged total staining demonstrating efficient coexpression, done as separate experiment from the surface staining. Insets are representative magnified images. Scale bar, $200 \mu \mathrm{m}$. $\boldsymbol{F}$, Histogram summarizing the quantification of surface labeled GluN2A in $\boldsymbol{E}$. ${ }^{* * *} p<0.001$; N.D., not determined because there was no signal. Sample size, $n=100$ cells.

terpreted at $3.4 \AA$. There are no significant differences between all three protomers. The refinement statistics are in Table 1.

\section{The structure of the GluN1-NTD protomer}

Similar to the crystal structures of other NTDs of glutamate receptor subunits, the GluN1-NTD protomer adopted a clamshell-like structure consisting of two globular subdomains, which we denote as R1 and R2 (Fig. 4A). The overall folding of the GluN1-NTD was similar to its homolog GluN2B-NTD (Fig. $4 A, B$ ). However, the GluN1-NTD contains a unique $\alpha$ helix (Fig. 
A

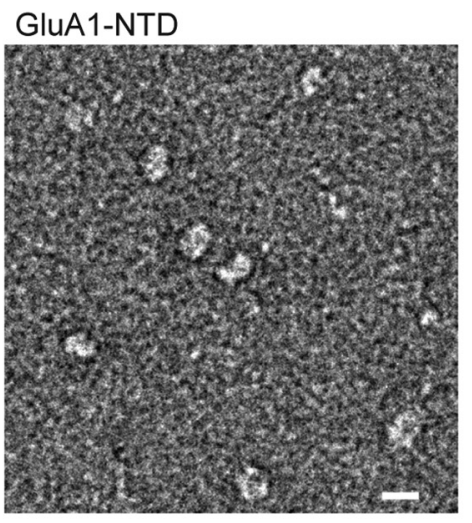

B
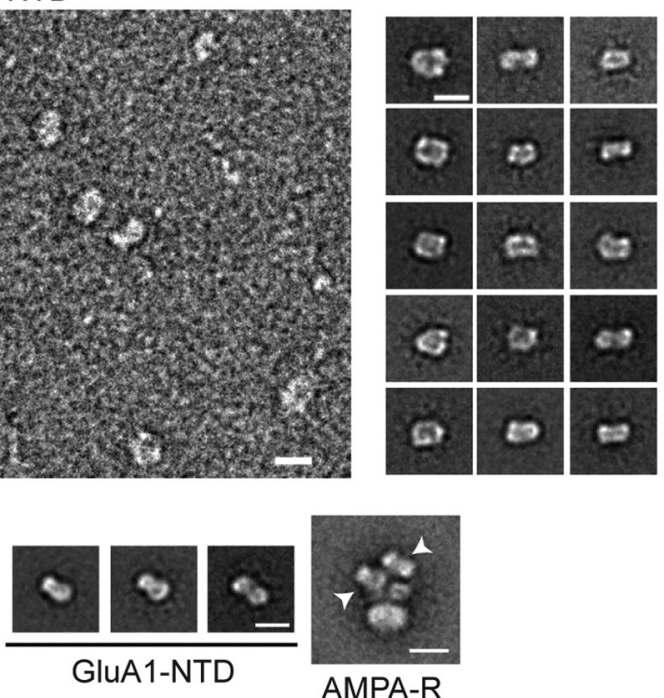

C
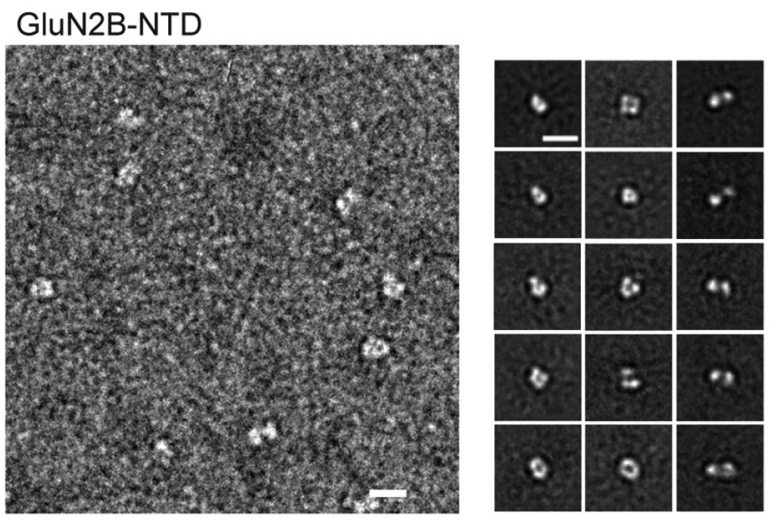

D
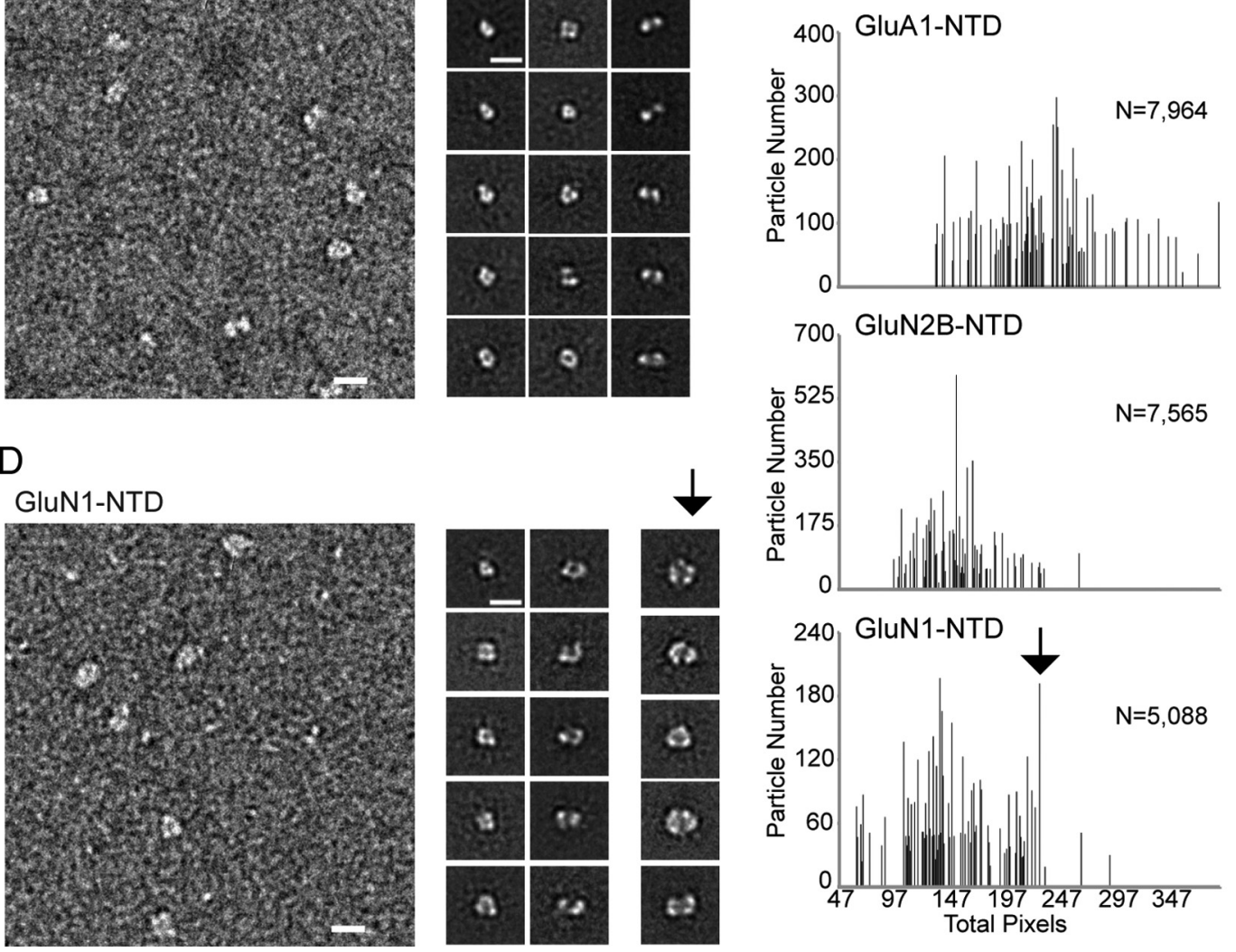

Figure 3. Single particle electron microscopy of the NTDs. A, Raw particle images of the GluA1-NTD (top left) and 15 representative class averages (top right). Bottom images are three representative class averages of the GluA1-NTD (bottom left) orientated similarly as the NTD of native tetrameric AMPA-R purified from rat brain (bottom right). Scale bar, $100 \AA$. $\boldsymbol{B}$, Histogram representing the number of particles in the categories defined below. The class averages of the GluA1-NTD were analyzed based on their ability to fill boxes of defined dimensions based on four different orientations, labeled I-IV, of the crystal structure of the GluA2-NTD (Protein Data Bank identifier 3H5V; bottom of histogram). Particles that cannot be unambiguously assigned to the categories were indicated as nonclassifiable (NC). I, $100 \times 95 \AA$ box; II, $100 \times 60 \AA$; III, $60 \times 60 \AA$ box; and IV, $60 \times 75 \AA$. Scale bar, $100 \AA$. C, Raw particle images of the GluN2B-NTD (left) shown with 15 representative class averages (right). Scale bar, $100 \AA$. D, Raw particle images of the GluN1-NTD (left) shown with 15 representative class averages (right). Arrow indicates larger particles seen in the class averages. Scale bar, $100 \AA$. $E$, Histograms summarizing the number of particles having the pixel area indicated in the horizontal axis. NTDs of GluA1 (top), GluN2B (middle), and GluN1 (bottom) were analyzed. Note the extra peak in the particles of the GluN1-NTD (indicated by arrow). Total particles analyzed were as follows: GluA1-NTD, $n=7964$; GluN1-NTD, $n=5088$; and GluN2B-NTD, $n=7565$. 
A

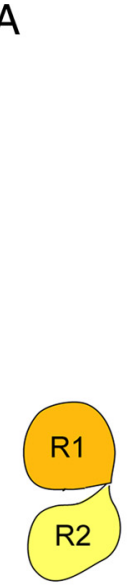

C

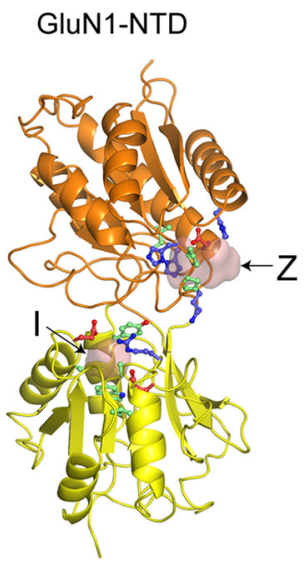

GluN1-NTD

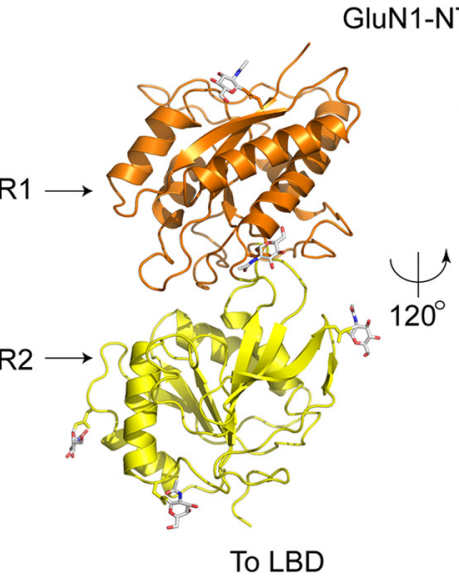

To LBD

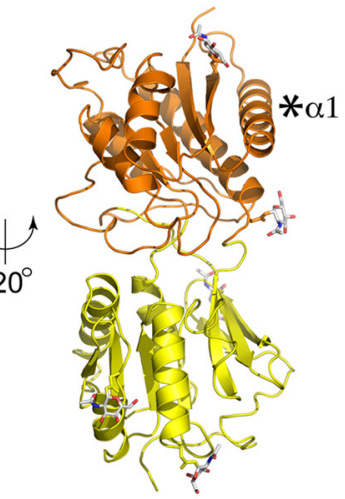

D
B

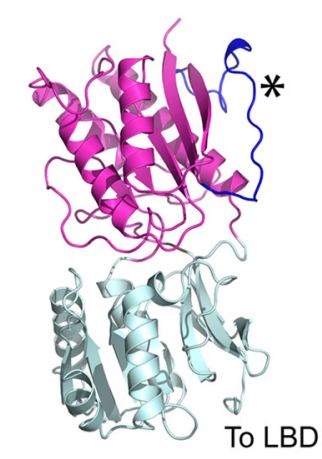

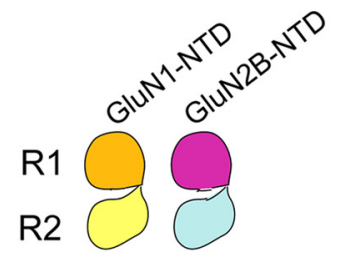
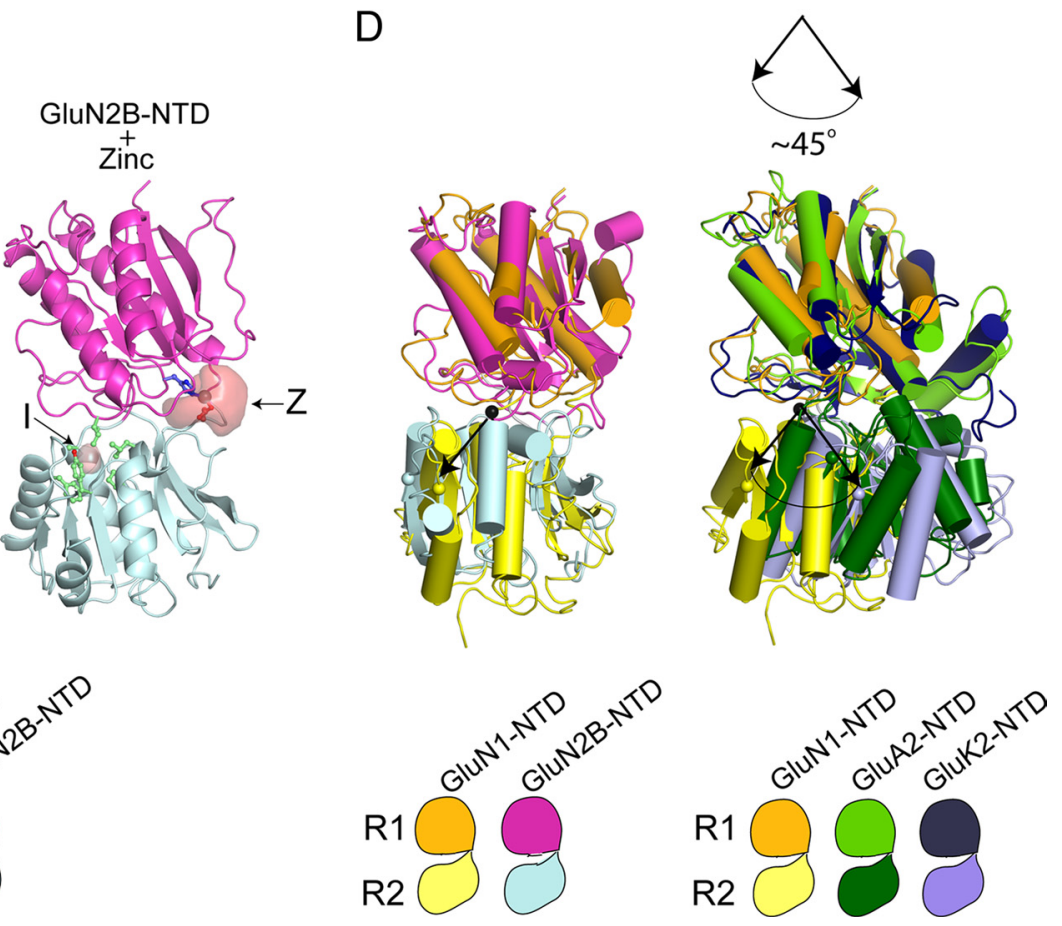

Figure 4. The crystal structure of a protomer of the GluN1-NTD. A, Single protomer of the GluN1-NTD viewed from two angles. The R1 (orange) and R2 (yellow) subdomains are indicated by arrows. A diagram of the clamshell-like arrangement of the subdomains is shown in the left bottom. Gray-colored atoms represent the five identifiable $N$-linked glycosylations (at residues N61, N203, N239, N276, and N300). B, The GluN2B-NTD (Protein Data Bank identifier 3JPW) shown in the same viewing orientation as the GluN1-NTD in $\boldsymbol{A}$ (right). Magenta, R1; light blue, R2. Asterisk (A, right) indicates $\alpha$ helix $1(\alpha 1)$ in the R1 subdomain of the GluN1-NTD that is an ordered coil in the GluN2B-NTD (asterisk and coil in blue). C, The cavities of the ifenprodil binding pocket (I) or zinc binding pocket (Z) in the zinc-bound GluN2B-NTD (Protein Data Bank identifier 3JPY, right) and the corresponding regions in the GluN1-NTD (left, indicated respectively as I and Z) are shown in red space fill. The two NTDs are shown from the same viewing angle. The local chemical environment of the pockets is shown by color coding amino acid residues (red, Asp and Glu; blue, Lys, Arg, and His; light green hydrophobic and aromatic, Phe, Met, Ala, Val, Ile, Leu). In C and $\boldsymbol{D}$ the color code used to represent the R1 and R2 domains of each NTD is also applied in the diagrams at the bottom. D, The GluN1-NTD shares the same twisted conformation as the GluN2B-NTD. The NTDs indicated at the bottom are superimposed after aligning the R1 subdomains. The "twisted" conformation was defined by the angle made between the pivotal point of rotation of the GluN1-NTD (Pro143; black sphere) to the end of $\beta 7$ in the R2 domain of each protomer. The R2 subdomains of the NTDs of the GluA2 and GluK2 are rotated around the pivot point by $45^{\circ}$ compared to the GluN1-NTD (arrows).

$4 A$, right, $\alpha 1)$ in the R1 subdomain that is absent in the GluN2BNTD (Fig. 4B, asterisk) (Karakas et al., 2009).

The $\mathrm{Zn}^{2+}$ ion binds to the NTDs of GluN2 subunits, inhibits channel function, and affects synaptic plasticity (Rachline et al., 2005; Izumi et al., 2006). Binding of ifenprodil to the GluN2BNTD also blocks channel activity. In contrast, no ligand is known to bind to the GluN1-NTD and modulate receptor function. The critical residues in the GluN2B-NTD that are required for ifenprodil binding reside in a hydrophobic pocket in the clamshell cleft (Karakas et al., 2009). In contrast, in the analogous pocket of GluN1-NTD most of the residues were polar or charged (Y144, W151, E172, L221, R252, E253) (Fig 4C, left, pocket "I"). The pocket of the GluN1-NTD in the analogous position as the $\mathrm{Zn}^{2+}$ binding pocket in the GluN2B-NTD contained hydrophilic residues (Fig. 4C, left, pocket "Z"). These observations raise the possibility that unidentified polar or charged molecules may act as ligands for the clamshell cleft of the GluN1-NTD.

When the R1 subdomain of the GluN1-NTD was aligned and superimposed on the NTDs of AMPA and kainate receptors, the R1 and R2 subdomains of the GluN1-NTD were rotated by $45^{\circ}$ relative to each other (Fig. $4 D$, right). A similar rotation was reported in the crystal structure of the GluN2B-NTD in its free and $\mathrm{Zn}^{2+}$-bound forms (Karakas et al., 2009), and thus the entire domain of the GluN1-NTD and GluN2B-NTD superimposed 
A

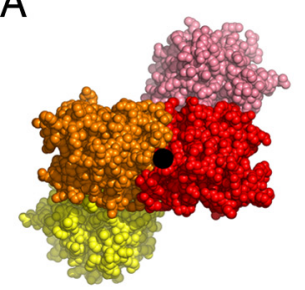

B

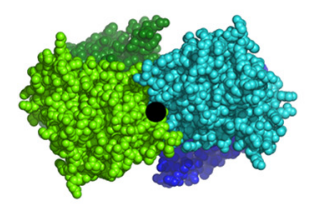

C

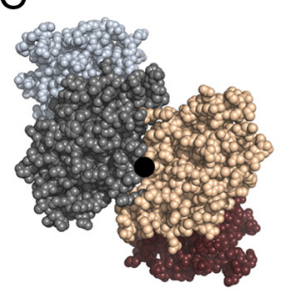

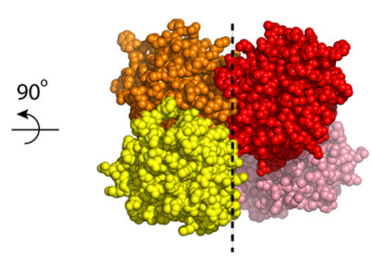
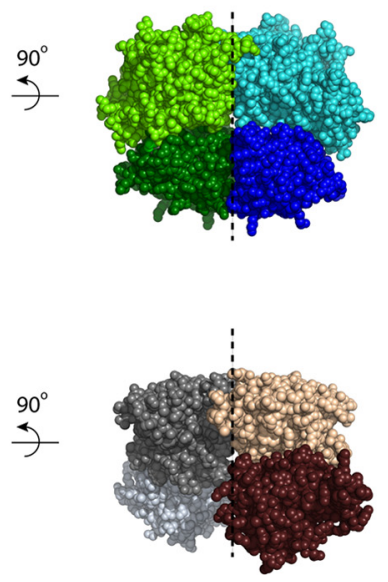
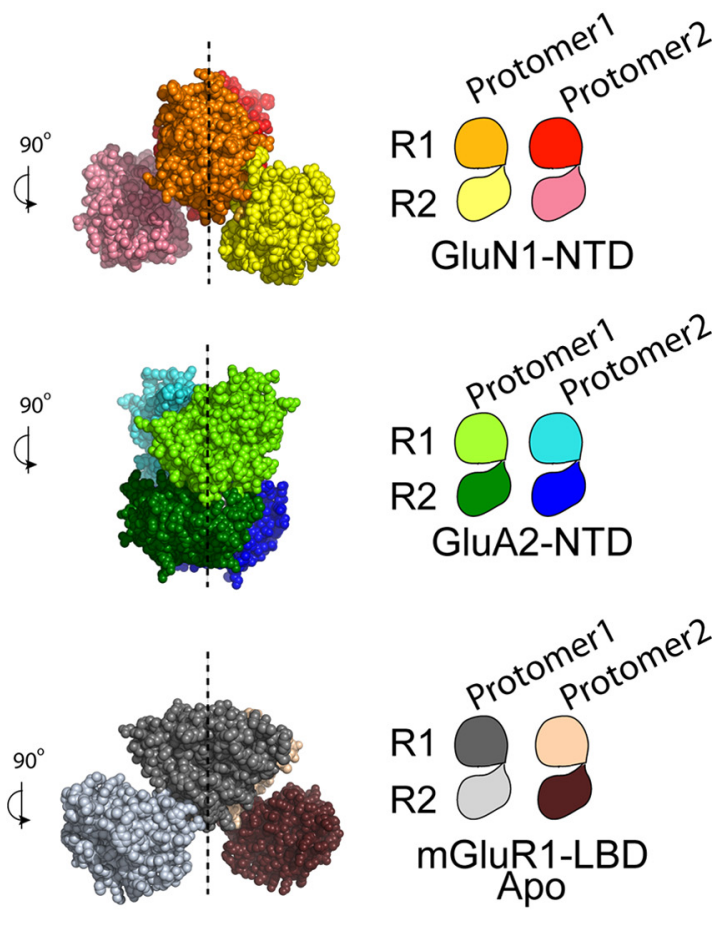

Figure 5. The structure and domain organization of the GluN1-NTD dimer and its homologs. A, GluN1-NTD viewed from three different orientations. The global twofold rotational axis of the dimer is shown as a dot in the top view (left) and as broken lines in the side views (middle and right). Orange R1 and yellow R2 subdomains form a protomer, while red R1 and pink R2 subdomains form another (also shown as diagram in the right). Note that the R2 portion (yellow and pink) is separated and does not contribute to the dimer interface. B, The GluA2-NTD dimer (Protein Data Bankidentifier $3 \mathrm{H} 5 \mathrm{~V}$ ) viewed as in $\boldsymbol{A}$. The global twofold rotational axis is shown as in $A$. Light green R1 and dark green R2 subdomains form a protomer, while light blue R1 and dark blue R2 subdomains form another (also shown as diagram in the right). $C$, The mGluR1-LBD (Apo-state) dimer viewed as in $\boldsymbol{A}$ and $\boldsymbol{B}$ (Protein Data Bank identifier 1EWT). Amino acids $344-407$ and $51-73$ were removed to better view the dimer interface. The global twofold rotational axis is shown as in $A$. Dark gray R1 and light gray R2 subdomains form a protomer, while light brown R1 and dark brown R2 subdomains form another (also shown as diagram on the right).

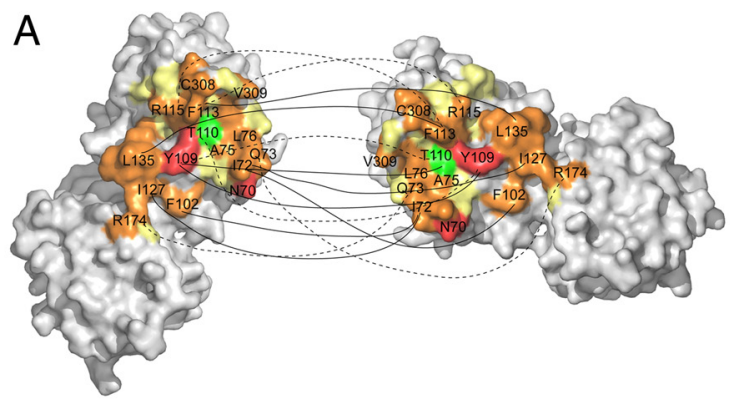

B
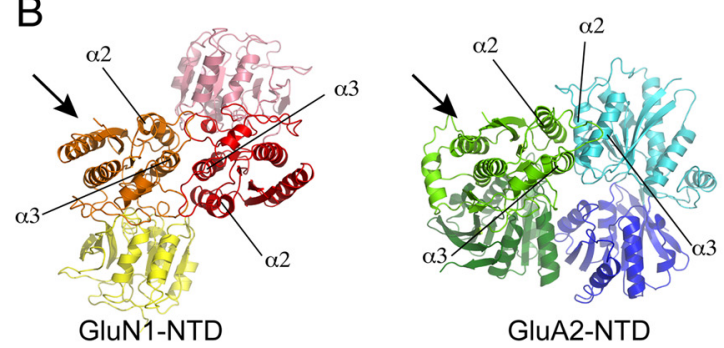

GluA2-NTD

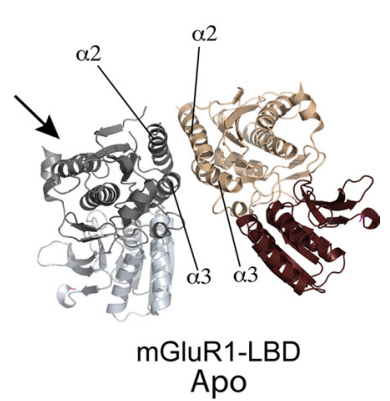

Figure 6. The dimer interface of the GluN1-NTD dimer. A, Surface representation of the two protomers of the GluN1-NTD that form a dimer. The colored surface represents residues in the buried dimer interface. Red and orange represent residues within the interface that make specific side chain interactions. Residue contacts are represented as connected lines. Solid black lines represent hydrophobic side chain interactions, while dotted lines represent polar or ionic interactions. Red (Y109) and green (T110) represent residues that were mutated in this study. The total buried surface area of the GluN1-NTD at the dimer interface has $\sim 1000 \AA^{2}$, which is comparable to the mGluR1-LBD $\left(\sim 900 \AA^{2}\right.$ ) (Kunishima etal., 2000) butnot as large as the GluK2 (Kumar etal., 2009) and GluA2-NTD (Jin etal., 2009) ( 1500 $\AA^{2}$ ), whose dimer interfaces are equally distributed among the R1 and R2 domains. $B$, The dimer structures of the GluN1-NTD, GluA2-NTD (Protein Data Bank identifier 3H5V), and mGluR1-LBD (Protein Data Bank identifier 1EWT) are shown after aligning one of the R1 subdomains of each structure. Arrow indicates the aligned R1 subdomain. The R1 and R2 subdomains of each NTD are color-coded as in Figure 5. The helices $\alpha 2$ and $\alpha 3$ are labeled. Note the relative orientation of the R2 domains is unique in each NTD dimer. As a result, the global positioning of the R1 subdomains is highly divergent in each NTD dimers. very well upon alignment (root mean square value $(\mathrm{RMS})=2.8 \AA$ ) (Fig. $4 D$, left). In contrast, the RMS values obtained from aligning GluN1-NTD with GluA2-NTD and GluK2-NTD were 3.82 and $3.39 \AA$, respectively. Because a single protomer of the GluN1-NTD structure resembles the ligand bound structure of the mGluR1-LBD $(\mathrm{RMS}=2.28 \AA)$ and the $\mathrm{Zn}^{2+}$ bound GluN2B-NTD structures (Karakas et al., 2009), the current GluN1-NTD structure may represent a closed clamshell conformation. This may suggest that in the form of an isolated domain the GluN1-NTD prefers to sample the closed cleft conformation, although it does not exclude the possibility that the clamshell stays open in the presence of other allosteric constraints in the context of mature NMDA-Rs.

The structure of the GluN1-NTD dimer The most striking feature of the crystal is the dimer made between the R1 subdomains of two protomers (Fig. 5A). The arrangement of the R1 and R2 subdomains of the two protomers was unique among the crystal structures of its homologs, namely the NTDs of GluA2 (Jin et al., 2009) (Fig. 5B) and the LBD of the mGluR1 (Kunishima et al., 2000) (Fig. 5C). The two R2 subdomains in the GluN1-NTD dimer extrude in oppo- 
A

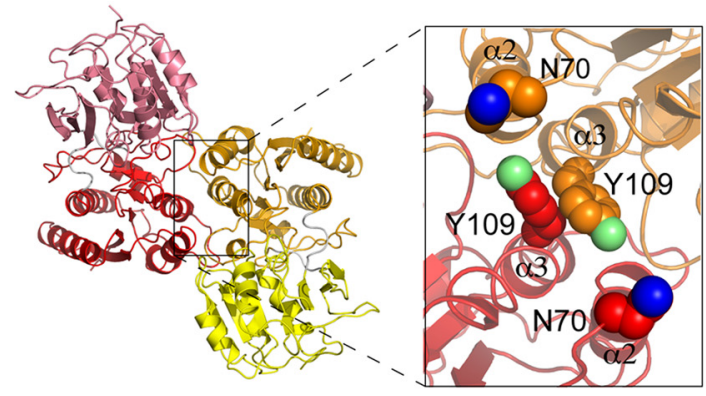

B
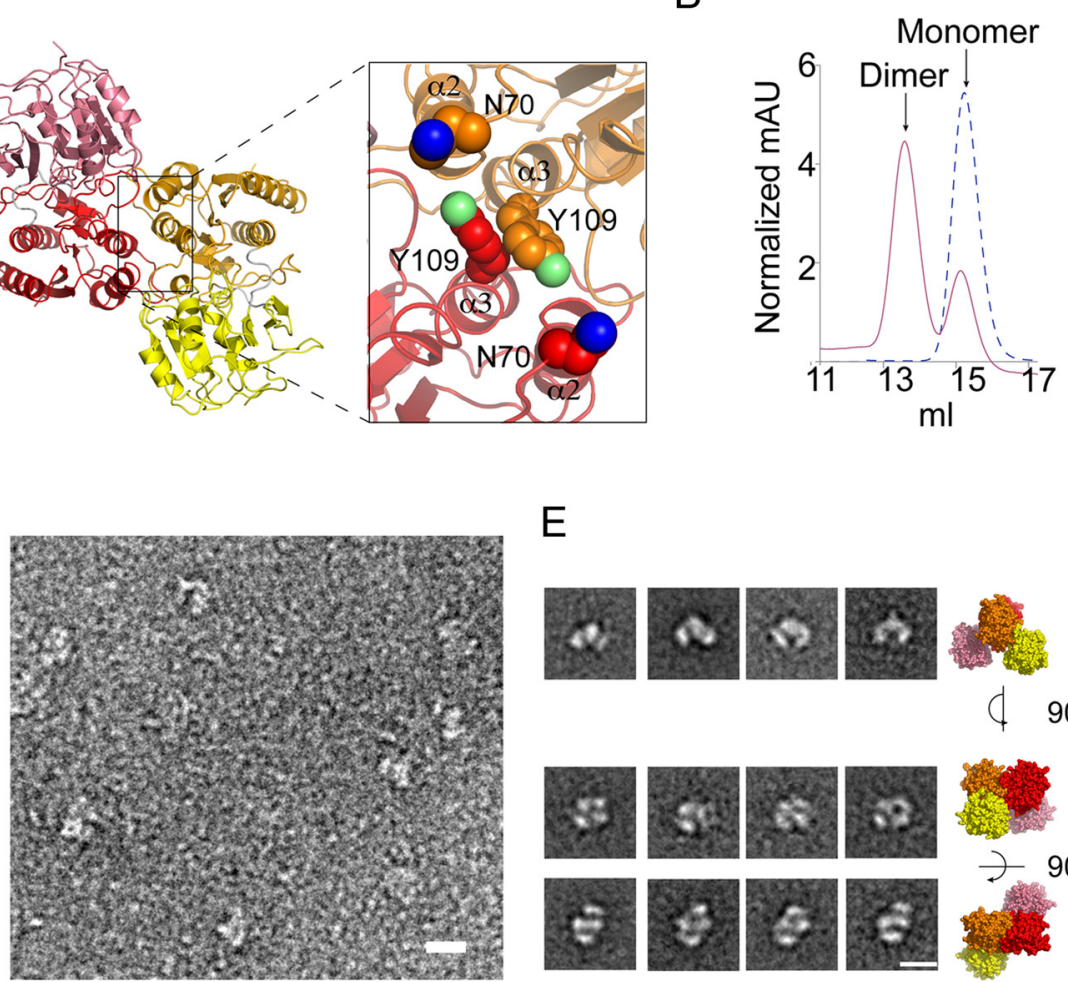

$E$

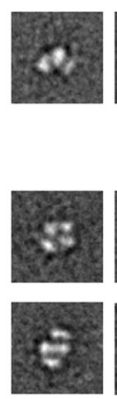

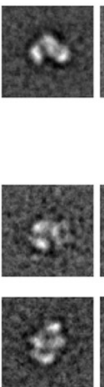

C

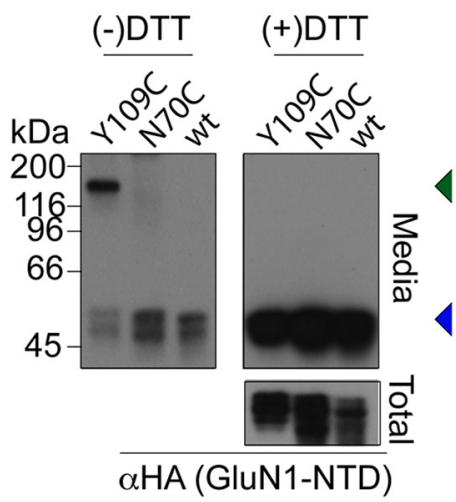

$\mathrm{F}$

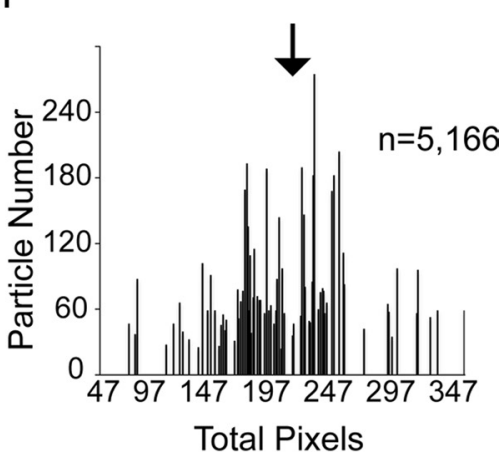

Figure 7. The $\mathrm{Y} 109$ C mutation stabilizes the GluN1-NTD dimer by forming an intermolecular disulfide bond. $A$, Diagram representation of the GluN1-NTD viewed from the bottom (the side where the LBD would be located) of the dimer (left). Magnification of the GluN1-NTD dimer interface centered at $\alpha$ helices 2 and 3 (right). Residues Y109 and N70 are represented as spheres. $\boldsymbol{B}$, Representative gel filtration chromatographs of the GluN1-NTD Y109C (solid red line) and wild type GluN1-NTD (dotted blue line). The monomer and dimer peaks are indicated by arrows. C, Western blots of the wt and mutant GluN1-NTD (N70C and Y109C). The SDS-PAGE was done under reducing [(+)DTT)] or nonreducing [(-)DTT] conditions. The left bottom panel is the Western blot of the total cell lysates of each protein after reducing SDS-PAGE. The green and blue filled arrowheads indicate the mobility of the GluN1-NTD homodimer and the GluN1-NTD monomer, respectively. Anti-HA $(\alpha H A)$ antibody was used as probe. D, Raw particle EM images of the GluN1-NTD-Y109C dimer. $\boldsymbol{E}$, Representative class averages of GluN1-NTD-Y109C. The projections structures in each row agree with the views of the crystal structure shown at the right end. $\boldsymbol{D}, \boldsymbol{E}$, Scale bar, $100 \AA$. $\boldsymbol{F}$, Histogram summarizing the number of GluN1-NTD-Y109C particles having the pixel area indicated in the horizontal axis. The arrow indicates the position of the peak in the particles of the wild-type GluN1-NTD (identical to the arrow shown in the bottom histogram of Fig. 3E). Total particles analyzed were $n=5166$.

site directions relative to the $\mathrm{R} 1$ subdomains. This global domain arrangement differs from the LBD dimer of mGluR1, in that protomers of the GluN1-NTD dimer are rotated in the opposite direction relative to protomers of the mGluR1-LBD dimer (Fig. 5, compare $A$, left, $C$, left). Side chain contacts at the GluN1-NTD are predominantly hydrophobic and occur between the $\alpha 3-\alpha 3$, $\alpha 2$-hyper variable loop (HVL) 2, and HVL2-HVL3. The details of the residue contacts are summarized in Figure $6 \mathrm{~A}$. The residues making contacts between the protomers in the GluN1-NTD dimer are located exclusively in the R1 subdomain and not in the $\mathrm{R} 2$, analogous to the LBD dimer of mGluR1 but distinct from the NTD dimers of GluA2 (Jin et al., 2009) and GluK2 (Kumar et al., 2009), which utilize both subdomains to form their dimer interfaces. In addition, the dimer interfaces formed by the R1 subdomain are derived from distinct sets of secondary structures in different NTD homologs, indicating diversity in the interactions among the R1 subdomains. This variety is obvious when we compare the different NTD dimer structures side by side after aligning the R1 subdomains of one of the protomer within each dimer (Fig. 6B, arrow indicates the aligned $\mathrm{R} 1$ subdomain). The contacts made in the asymmetric unit between the third protomer and the dimer described above were not investigated further, since the assembly was unlikely to be biologically relevant.

\section{The Y109C mutation induces stable NTD dimers}

What is the functional relevance of the dimeric domain arrangement observed in the crystal structure of the GluN1-NTD? We reasoned that the homodimeric arrangement of the GluN1-NTD should be a permissive conformation of the mature NMDA-Rs if the receptors could assemble correctly while constraining the GluN1-NTDs into dimers. Alternatively, if the dimerization of the GluN1-NTD is only permissive during the assembly process and unfavored in the mature NMDA-Rs, constraining the GluN1-NTD into dimers will prevent the GluN1 subunit from assembling into the tetrameric NMDA-Rs. To manipulate the dimerization state of the GluN1-NTD, we engineered the residues in the homodimer interface.

Tyrosine 109 (Y109) resides in the center of the dimer interface and is located at an ideal distance $(\sim 3.4 \AA)$ to form a disulfide bond between the two protomers (Fig. $7 A$ ). In fact, when the Y109C mutation was introduced, $80 \%$ of the mutant NTD was a stable dimer as determined by gel filtration chromatography, whereas the majority of the wild-type GluN1-NTD was monomeric in solution (Fig. 7B). The GluN1-NTD-Y109C dimer was disrupted upon treating the protein with the reducing reagent DTT, as demonstrated by the shift of mobility of the GluN1NTD-Y109C mutant in SDS-PAGE (Fig. 7C). To test the specificity of cysteine-induced dimer formation, we investigated another mutant: GluN1-NTD-N70C. The N70 residue is positioned in the dimer interface in the neighborhood of Y109, however in an opposite direction along the interface. Thus, even if a cysteine was introduced in the N70 position, the two cysteines would be too far to form a disulfide bond in the homodimer (Fig. 
A

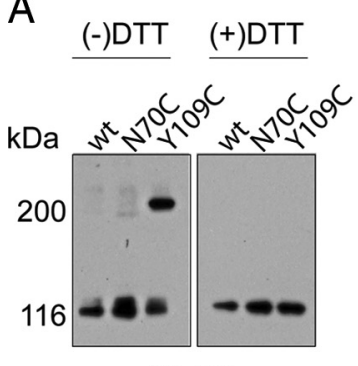

$\alpha$ GluN1
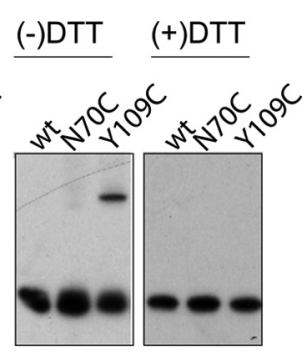

$\alpha$ GluN1

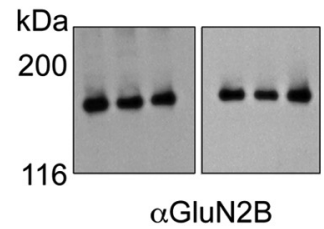

C

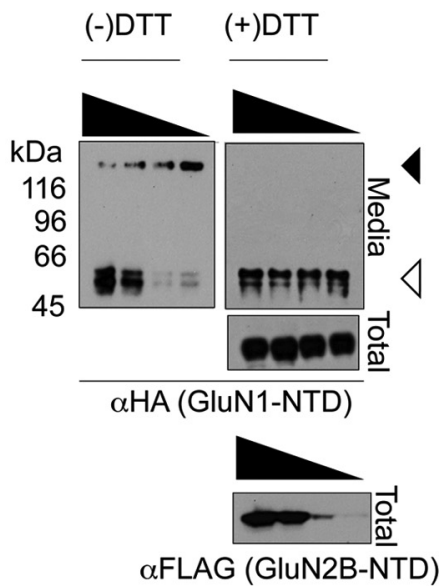

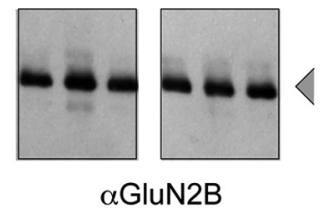

B

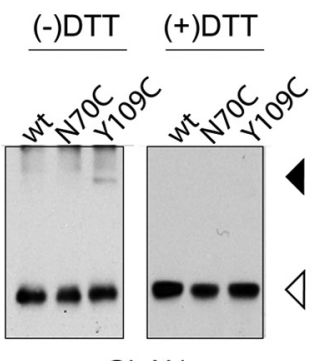

$\alpha$ GluN1

$\alpha$ GluN2B

$\mathrm{D}$

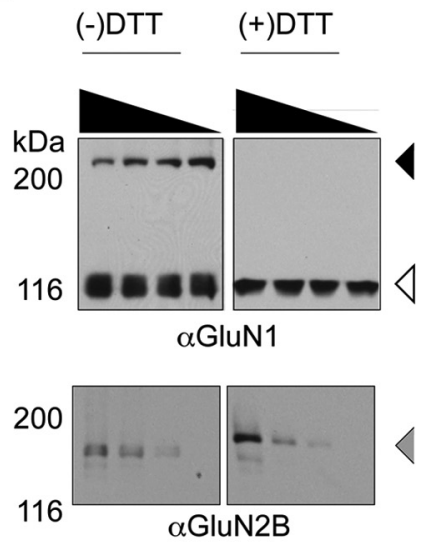

Figure 8. The effect of $\mathrm{Y} 109 \mathrm{C}$ mutation on the assembly of heterotetrameric NMDA-Rs. $A$, Western blots of the total lysates of HEK-TetON cells. Each sample was resolved by SDS-PAGE under reducing [(+)DTT and nonreducing (-)DTT] conditions. Left pair, Total lysates from cells singly expressing GluN1 variants [wt or mutants (Y109C and N70C)]. Right pair, Total lysates from cells dually expressing GluN1 variants and wild-type GluN2B. In $\boldsymbol{A}-\boldsymbol{D}$, the antibodies used in each Western blot are indicated in the bottom of each panel. In $\boldsymbol{A}, \boldsymbol{B}$, and $\boldsymbol{D}$, the black-filled and open arrowheads indicate the positions of the GluN1 homodimer and monomer, respectively. The gray-filled arrow indicates the position of the GluN2B monomer. $\boldsymbol{B}$, Western blots of the complex made of GluN2B and GluN1 variants [wt or mutants (Y109C and N70C)]. As above, the SDS-PAGE was done under both reducing and nonreducing conditions. The GluN1 variants and GluN2 were expressed using a dually expressing plasmid, and the complex was immunoprecipitated using the anti FLAG antibody that recognizes the FLAG tag of the GluN2B subunit. C, Western blots of culture supernatant (Media) of GnTI(-) HEK cells cotransfected with a fixed amount of plasmid expressing GluN1-NTD-Y109C and increasing amounts of plasmids expressing GluN2B-NTD-FLAG (indicated by the filled triangles). In each condition the total transfected plasmid was made equal by supplementing the DNA quantity with an empty plasmid. As above, the SDS-PAGE was done under both reducing and nonreducing conditions. The left bottom panels (Total) are the Western blot of the total cell lysates of each protein after the reducing SDS-PAGE. The black-filled and open arrowheads indicate the positions of the GluN1-NTD dimer and monomer, respectively. Anti-HA and anti-FLAG antibodies were used to probe for GluN1-NTD and GluN2B-NTD, respectively. $D$, Western blots of total cell lysates from HEK-TetON cells cotransfected with a fixed amount of plasmid expressing GluN1-Y109C and increasing amounts of plasmids expressing FLAG-GluN2B (indicated by the filled black triangle). In each condition the total transfected plasmid was made equal by supplementing the DNA quantity with an empty plasmid. As above, the SDS-PAGE was done under both reducing and nonreducing conditions. The positions of the monomers and dimer are indicated by the arrowheads as in $\boldsymbol{A}$ and $\boldsymbol{B}$.

$7 A)$. Consistently, the disulfide-mediated dimerization was not observed in GluN1-NTD-N70C (Fig. 7C), suggesting that the disulfides formed in the dimeric GluN1-NTD-Y109C are specific. Because N70 is located on $\alpha 2$ helix, the lack of disulfide formation in N70C mutation also confirms that the homodimerization of GluN1-NTD does not use the interactions between the two $\alpha 2$ helices such as seen in the homodimers of mGluR1-NTD.

The single particle EM projection structures of the negatively stained GluN1-NTD-Y109C mutant were consistent in size and shape with the views of the dimeric crystal structures of the GluN1NTD (Fig. $7 D-F$ ). We also noted that in the wild-type GluN1-NTD, $5 \%$ of the particles adopted the size and shapes that were in agreement with a dimer and resembled the projections of the GluN1NTD-Y109C (Fig. 3, compare $D$, arrow, $E$, second row). Collectively, these data suggest that the GluN1-NTD-Y109C mutation forces the majority of themselves into dimers such as seen in the crystal structure by forming a disulfide bond between the protomers.

\section{The effect of Y109C mutation on subunit assembly}

Can the NTDs of GluN1 subunit homodimerize in the context of the fulllength subunit? The Y109C mutation was introduced into the full-length GluN1 subunit and expressed in HEK-TetON cells. After solubilizing the receptors from the membrane using the detergent dodecyl maltoside, their mobility in SDSPAGE was compared in the presence or absence of the reducing reagent DTT. If disulfide-mediated cross-linking of the NTDs occurred, GluN1 should have a mobility twice its calculated molecular weight of $100 \mathrm{kDa}$ in the absence of DTT. In fact, the GluN1-Y109C migrated at 100 $\mathrm{kDa}$ in the presence of DTT but shifted to $200 \mathrm{kDa}$ under the nonreducing condition (Fig. $8 \mathrm{~A}$, left). On the other hand, the wild-type GluN1 and GluN1-N70C both migrated at their calculated molecular weight of $100 \mathrm{kDa}$ under both reducing and nonreducing conditions, suggesting that the disulfide cross-linking observed in the GluN1-Y109C is specific. Collectively, these results suggest that the dimerization of the GluN1-NTD, such as seen in the crystal structure, is permissive in the context of the intact GluN1 subunit dimer.

Next, we tested whether NMDA-Rs made of GluN1 and GluN2B subunits can assemble in the presence of the GluN1Y109C mutation. For this purpose both the GluN1-Y109C and wild-type GluN2B were coexpressed in HEK-TetON cells, solubilized in dodecyl maltoside as described above, and their mobility in the SDS-PAGE was examined in the presence or absence of DTT. The GluN1-Y109C in the total extract was able to crosslink even in the presence of the GluN2B subunit, while the wild type and the GluN1-N70C remained as monomers [Fig. $8 A$, right top, $(-)$ DTT and $(+)$ DTT]. In these experiments the coexpressing wild-type GluN2B subunit was detected only at its calculated molecular weight of $180 \mathrm{kDa}$ in both reducing and nonreducing conditions (Fig. $8 \mathrm{~A}$, right bottom). However, when we examined the GluN1-Y109C that coimmunoprecipitated with the GluN2B subunit, only a small fraction of the GluN1-Y109C was cross- 

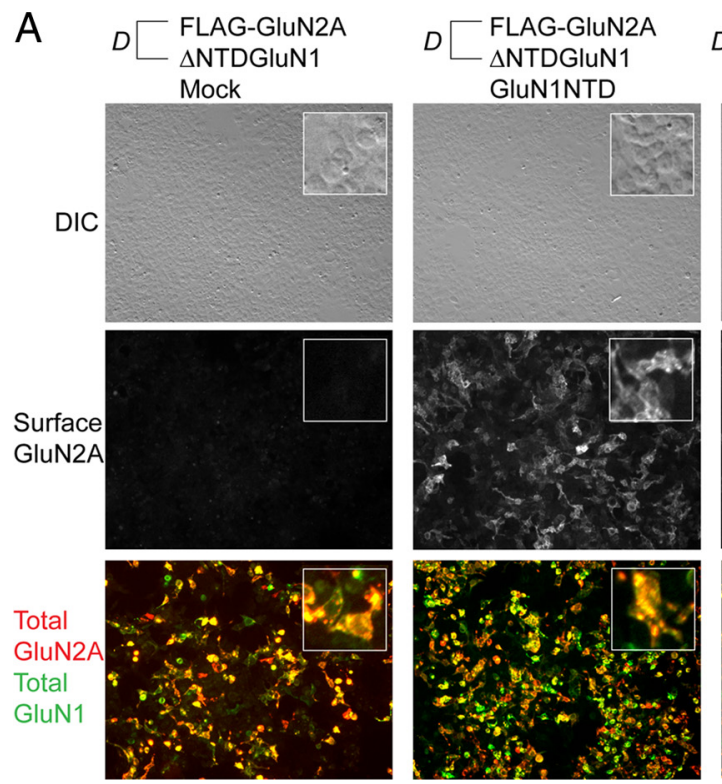
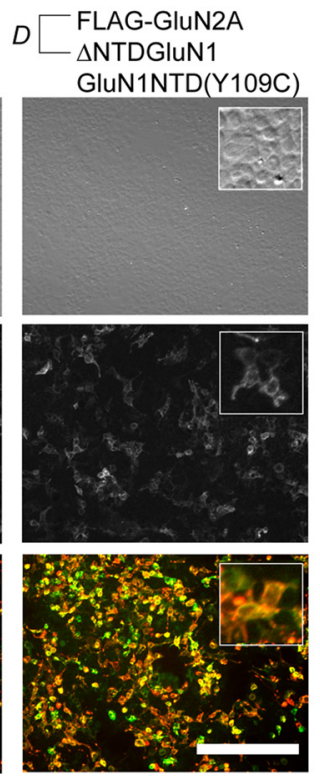

B

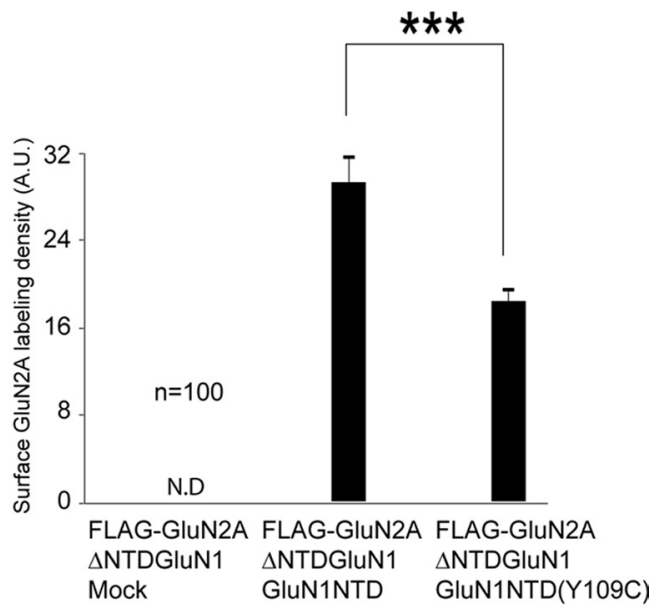

Figure 9. The effect of $\mathrm{Y} 109$ ( mutation of GluN1 on receptor maturation. $A$, Images of HEK-TetON cells cotransfected with plasmids that express the proteins indicated at the top of each column. " $D$ " indicates that the two proteins were expressed using a dual expressing plasmid. The empty plasmid without an insert was used in the left column as negative control (Mock). Top row, Differential contrast interference (DIC) image. Second row, Surface staining of the identical field using an anti-FLAG antibody that detects the full-length FLAG-GluN2A on the cellular surface. Bottom row, The merged total staining demonstrating efficient coexpression done as a separate experiment as the surface staining. Insets are representative magnified images taken from the same field. Scale bar, $200 \mu \mathrm{m} . \boldsymbol{B}$, A histogram summarizing the quantification of the cell surface GluN2A signal in $\boldsymbol{A} .{ }^{* * *} p<0.001$; N.D, not determined because there was no signal. Sample size, $n=100$ cells.

linked by the disulfide bond (Fig. 8 B). No cross-linking was observed in the wild type nor N70C (Fig. 8 B). These results indicate that the forced dimerization of the GluN1-NTD is unfavorable in the mature heteromeric NMDA-Rs. Similar results were obtained when we conducted the same experiments but used GluN2A instead of GluN2B (data not shown).

\section{The GluN2-NTD competes with homodimerization of GluN1-NTD}

The observation that GluN1 can form dimers through their NTDs and, at the same time, the stable NTD dimerization is unfavorable for forming a complex with GluN2 suggests the possibility that the separation of the GluN1-NTD dimers is required for progression through the receptor assembly pathway. If this is the case, the disulfide cross-linking of the GluN1-NTD-Y109C should be inhibited by the presence of excess GluN2-NTD. To alter the relative quantity of GluN1-NTD-Y109C and GluN2NTD in the cell, we cotransfected a fixed amount of plasmid that expresses GluN1-NTD-Y109C with varying quantities of plasmid that expresses GluN2-NTD. In each condition GluN1-NTDY109C expressed at the same level, and the expression level of GluN2-NTD correlated with the amount of plasmid that was cotransfected, as determined by the reduced SDS-PAGE with DTT (Fig. 8C, (+)DTT). In the nonreduced SDS-PAGE, however, we found that the amount of high molecular weight crosslinked species of GluN1-NTD-Y109C was inversely proportional to the quantity of the coexpressed GluN2-NTD (Fig. 8C, $(-)$ DTT). Similar results were obtained when we conducted the experiment using the full-length subunits (Fig. 8D). These results suggest that homodimerization of the NTD of GluN1 subunit was dose-dependently inhibited (or competed) by the GluN2 subunit, consistent with the idea that separation of the GluN1-NTD dimer is necessary for the heteromeric assembly of NMDA-Rs.

\section{Effect of the Y109C mutation on receptor trafficking}

As described earlier (Fig $1 B, C$ ), when $\Delta$ NTD-GluN1 and GluN2 were coexpressed, GluN2 cannot reach the surface. This defect was rescued by further coexpressing GluN1-NTD. Using the same assay, we tested whether the GluN1-NTD-Y109C can rescue the trafficking defect of GluN2 caused by $\Delta$ NTD-GluN1. In keeping with our model that the separation of the GluN1-NTD homodimer is necessary for subunit assembly, the surface expression of GluN2A was significantly decreased when GluN1-NTDY109C was coexpressed instead of wild-type GluN1-NTD (Fig. $9 A, B)$. A small amount of GluN2A reached the surface when the GluN1-NTD-Y109C was coexpressed, which we interpret as a contribution of the minority of GluN1-NTD-Y109C that did not form covalent homodimers (Fig. $7 B$, red trace). Because promoting either heterodimer (Fig. 2E,F) or homodimer (Fig. 9) is unfavorable for surface expression, an optimal degree of homodimerization and heterodimerization of the NTDs is thus critical for efficient receptor assembly. Collectively, our results suggest that the initial homodimerization and subsequent heterodimerization are both required for efficient subunit assembly of NMDA-Rs.

\section{Discussion}

In this study we determined the biochemical characteristics, single particle EM structure, and crystal structure of the GluN1NTD. In solution, the GluN1-NTD exists as a mixture of monomer and dimer. The GluN1-NTD can also interact with the GluN2-NTDs, indicative of complex interdomain contacts among the NTDs. The interdomain contacts between the subunits contribute to subunit assembly and channel function and thus are critical for understanding the mechanism of NMDA-R function. By comparing the biochemical and cell biological phenotypes of wild-type and two mutant GluN1 subunits (T110A and $\mathrm{Y} 109 \mathrm{C}$ ) defective in receptor maturation, we revealed a molecular determinant of NMDA-R assembly.

The T110A mutation, identified through sequence analysis, promotes the heteromeric interaction between the NTDs of GluN1 and GluN2B. The dimeric crystal structure of the GluN1NTD revealed a novel homodimer interface, enabling us to interpret the underlying mechanism of T110A phenotype. T110 forms 


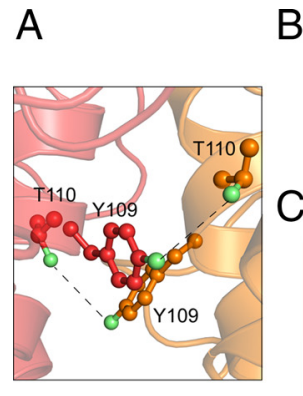

B

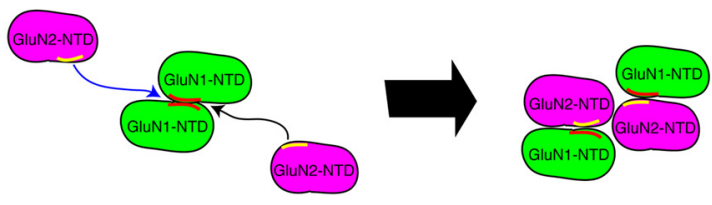

C
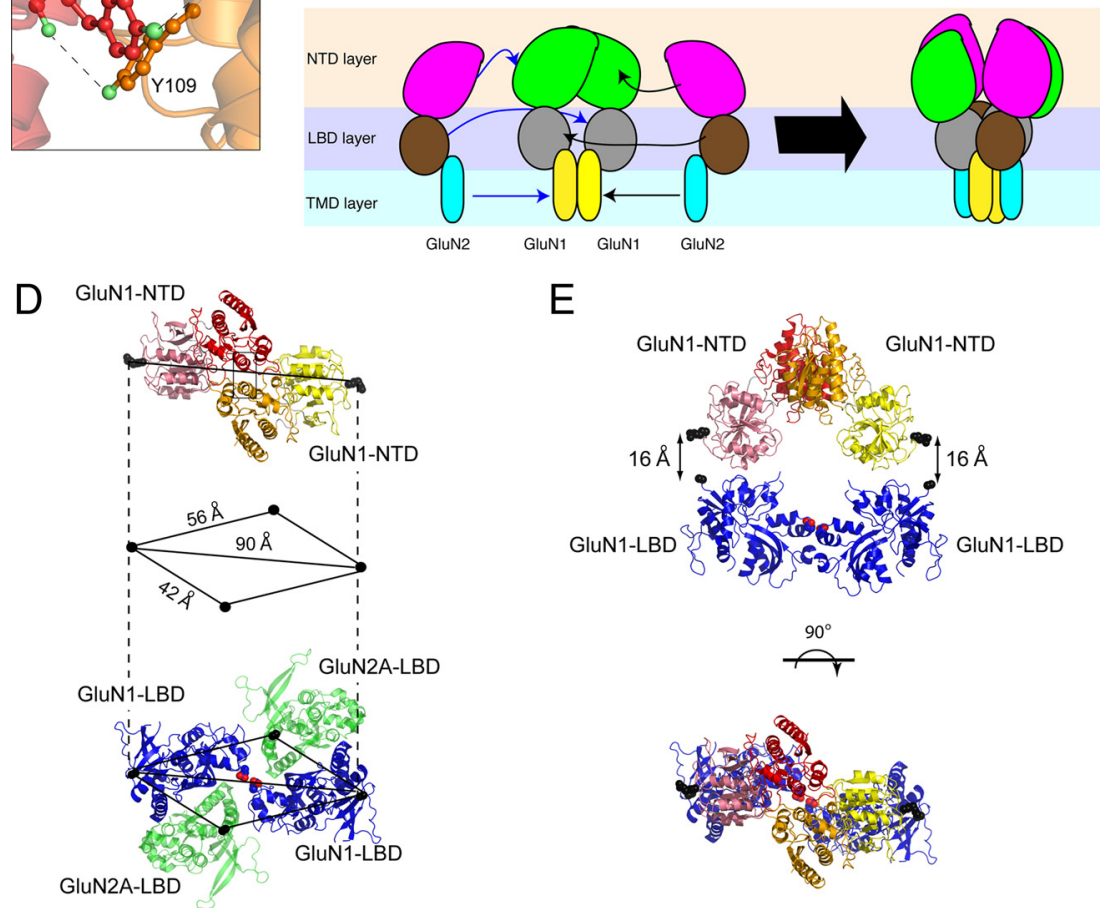

$\mathrm{E}$
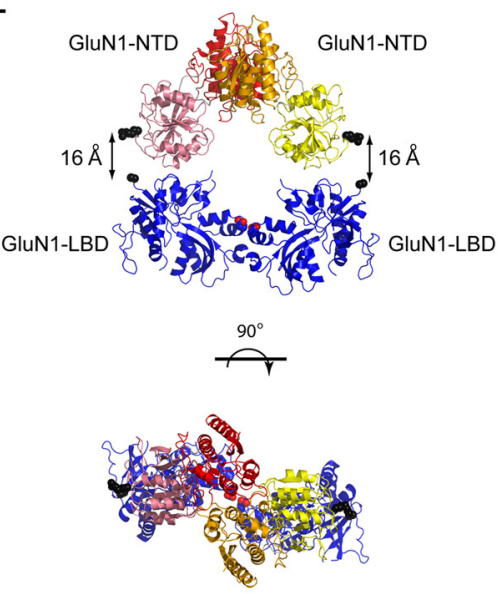

Figure 10. A model for the assembly pathway of heterotetrameric NMDA-Rs. $A$, Side chain interactions between $\mathrm{Y} 109$ and $\mathrm{T} 110$ at the GluN1-NTD dimer interface. Dotted line represents a hydrogen bond made between T110 and Y109. B, A model describing the rearrangement of the NTDs during the assembly of heterotetrameric NMDA-Rs. Left, Dimer of GluN1-NTDs and two monomers of GluN2-NTDs. Right, Putative heterotetramers of the NTDs in the mature NMDA-R. The initial homodimerization of the GluN1NTD is critical for the recruitment of the GluN2-NTD. Subsequent rupture of the GluN1-NTD homodimer facilitates tetramer formation. The red lines indicate the homodimer interface of GluN1-NTD. The yellow lines indicate the corresponding surface of the GluN2-NTD involved in interdomain contacts. C, A model depicting the subunit assembly pathway of the heterotetrameric NMDA-R. Left, Dimeric GluN1 and two monomers of GluN2. Right, Heterotetrameric NMDA-R. Each subunit is represented by the globular domains of NTD (green and magenta), LBD (gray and brown), and TMD (yellow and light blue). The domains are also positioned in "layers" as indicated in the background. The homodimer of GluN1 is initially formed and serves as a scaffold to recruit GluN2s. The switching of the interdomain contacts between the NTDs is critical for the receptor assembly. The LBDs form a pair of heterodimers according to Furukawa et al. (2005). The arrows indicate the interdomain interactions between the NTDs and the LBDs during tetramerization. $\boldsymbol{D}$, Comparison of the geometry between the GluN1-NTD dimer and proposed LBD heterotetramer (Sobolevsky et al., 2009). Top, GluN1-NTD dimer viewed from the LBD side. The black spheres are the C-terminal ends of each NTD. Bottom, Heterotetramer of the LBDs formed of GluN1 and GluN2A, viewed from the NTD side. The black spheres are the N-terminal ends of each LBD. Parallel solid and broken lines are drawn to indicate the alignment of the two structures. The distances between points are indicated. $\boldsymbol{E}$, The GluN1-NTD and GluN1-LBD in D were placed together so that the C termini of the NTDs and the N termini of the LBDs are in close proximity. Two different views are shown. The distances between the closest C-terminal of the NTD and the $\mathrm{N}$-terminal of the LBD is $16 \AA$.

an intermolecular hydrogen bond with Y109 (Fig. 10A). We predicted that Y109 is also stabilized by T110 and that replacing the T110 with alanine would eliminate the hydrogen bond and further rearrange the position of Y109. The T110A mutation may thus restructure the interface and potentially have adverse effect on homodimerization. The crystal structure also enabled us to identify the Y109C mutation that accelerates homodimerization of the GluN1-NTD via disulfide bonding. Importantly, Y109C and T110A promote homodimerization and heterodimerization, respectively, as a consequence of engineering the identical neighborhood within the homodimer interface of GluN1-NTD.

The wild-type GluN1 forms dimers (Atlason et al., 2007). We extend the previous finding by demonstrating disulfide bond forma- tion in the GluN1-Y109C dimer and determining that the dimerization occurs through the NTD. The dimerization through the Y109 was specific, as GluN1N70C had no effect. The GluN1-Y109C, however, did not assemble well with the GluN2 subunits, indicating that the homodimer interface of the GluN1-NTD must be available for the heteromeric assembly of NMDA-Rs. Consistently, manipulating the same homodimer interface in a nearby location by introducing the T110A mutation stabilized the heteromeric interaction between the NTDs of GluN1and GluN2. It is then conceivable that the identical surface of the GluN1-NTD is used for both homodimerization and heterodimerization. The promiscuity of the NTD interactions between the GluN1 and GluN2 can explain the different observations and models reported for the mechanism of NMDA-R assembly (Meddows et al., 2001; Qiu et al., 2005; Atlason et al., 2007; Schüler et al., 2008; Gielen et al., 2009; Sobolevsky et al., 2009).

Our data suggest that when GluN1 is synthesized alone it has the tendency to homodimerize through the NTDs (Fig. $8 A$ ). Previous EM studies have demonstrated that clusters of ribosomes are formed in the endoplasmic reticulum, suggestive of a polysome-like assembly (Palay and Palade, 1955; Peters et al., 1991). In these assemblies, multiple molecular copies of membrane proteins would be translated at a high local concentration. It is conceivable that under such an environment weak interdomain interactions, such as those seen in the GluN1-NTD homodimer, would have a significant impact and lead to homodimer formation of the GluN1 during the initial stage of subunit assembly.

GluN1 was more stable than GluN2 and was proposed to promote receptor assembly (Atlason et al., 2007). The initial homodimerization of the GluN1-NTD indeed appears to play an important role during the subunit assembly. If simply promoting the interaction between the GluN1-NTD and GluN2-NTD is sufficient for subunit assembly, one would expect that the T110A mutation should accelerate the process. However, compared to the wild type, the T110A only partially rescued the trafficking defect of GluN2 caused by the $\triangle$ NTD-GluN1. The GluN1-NTDT110A not only has increased affinity to the GluN2-NTD but also enhances the secretion of the GluN2-NTD. Despite the stronger inherent ability to promote the secretion of the GluN2-NTD, the GluN1-NTD-T110A cannot rescue the impaired surface expression of the NMDA-R caused by $\triangle$ NTD-GluN1. This observation further supports the requirement for the initial homodimerization of GluN1-NTD for efficient subunit assembly. Our results may imply that the homodimeric GluN1-NTD functions as a scaffold to assist the recruitment of the GluN2 subunit at a spatial 
geometry favorable for the heteromeric subunit assembly (Fig. $10 B, C)$. The subsequent rupture of the homodimer interface of GluN1-NTD may promote its interaction with the GluN2-NTD, resulting in the stabilization of the heteromeric receptor. Our study did not take into account the contribution of the other domains (the LBD, TMD, and CTD). However, it is known that the NTD and the TMD are dimerized during the subunit assembly process of the AMPA-Rs (Shanks et al., 2010). Based on the analogy to the subunit assembly mechanism of the AMAP-Rs, we speculate that the TMD of the GluN1 may also be dimeric.

Consistent with the function of the GluN1-NTD homodimer as a scaffold for subunit assembly, the homodimeric GluN1-NTD has the geometry compatible to connect with the tetrameric model of the LBDs (Sobolevsky et al., 2009) (Fig. 10 D, E). Specifically, when we compare side by side the GluN1-NTD dimer structure and the two LBD heterodimers made of GluN1 and GluN2A (Furukawa et al., 2005; Sobolevsky et al., 2009), the distance between the N termini of the LBDs of GluN1 ( $90 \AA$ ) is in keeping with the distance between the two $C$ termini of the GluN1-NTD dimers (Fig. 10D). The linker that connects the NTD and the LBD of GluN1 subunit consists of 18 aa. When we combine the crystal structures of the current GluN1-NTD and the known GluN1-LBD (Furukawa et al., 2005), six residues in the NTD-LBD linker are not resolved. In the combined model, the distance between the C terminus of the GluN1-NTD and the $\mathrm{N}$ terminus of the GluN1-LBD is predicted to be $16 \AA$ (Fig. $10 \mathrm{E}$, top), a distance that could be easily spanned by the unresolved six residues in the connecting linker. It is then conceivable that, in the homodimer of GluN1, the homodimeric NTDs will position the LBDs into the relative geometry seen in the mature tetramer. The LBD of GluN1 crystallizes as homodimer when bound to cycloleucine (Inanobe et al., 2005). The distance, however, between the two $\mathrm{N}$ termini of this LBD dimer is $45 \AA$, and thus geometrically inconsistent with the GluN1-NTD dimer reported in this study.

A small but significant amount of cross-linked GluN1-Y109C was able to assemble with the GluN2 subunit (Fig. 8B, Y109C). The geometric compatibility between the GluN1-NTD dimer and the LBD tetramer also raises the possibility that the GluN1NTD dimer is permissive in the mature NMDA-Rs under a certain conformational state. In this model, the NTDs connected to the two diagonally arranged GluN1-LBDs would have to form a homodimer, suggesting an unexpected complex connection between the domains that was not observed in the crystal structure of the tetrameric AMPA-R (Sobolevsky et al., 2009). Because the GluN1-NTD and GluN2-NTD were suggested to rearrange when the NMDA- $\mathrm{R}$ is blocked by $\mathrm{Zn}^{2+}$ and ifenprodil (Gielen et al., 2009), if the above conformation exists it would be consistent with the blocked state of the ion channel. It is then possible that homodimerization of the GluN1-NTD following the rupture of the NTD heterodimer would stabilize the receptor architecture. More data are needed to definitively answer these hypotheses. In conclusion, the GluN1-NTD dimer adopts a unique conformation that is different from the NTDs of the homologous AMPARs, proposing new hypotheses for investigating NMDA-R structure and function. The current data suggest that homodimerization and subsequent dissociation of the GluN1-NTD play critical roles during the subunit assembly process. This finding opens up a new venue in the inquest of the glutamate receptor assembly process. Detailed knowledge of this process is essential not only to understand the underlying mechanistic aspect of the channel, but also to design therapeutics controlling the activity of the channel.
Our results also highlight that the twisted interlobe NTD conformation (Fig. 4D) is a distinct property of NMDA-R subunits that is absent in AMPA/kainate receptor subunits (Clayton et al., 2009; Jin et al., 2009; Kumar and Mayer, 2010). This observation, together with others (Karakas et al., 2009; Stroebel et al., 2011), strongly suggests that the overall arrangement of the NTD layer differs between AMPA/kainate and NMDA receptors and raises the possibility that alternative models for NMDA-R architecture exists other than what was derived from extending the concepts obtained from results on AMPA-Rs (Sobolevsky et al., 2009).

The crystal structure also identified the binding pocket "I" in the GluN1-NTD whose surface is made of hydrophilic side chains. The analogous pocket "I" in GluN2B-NTD is largely hydrophobic and is the putative binding site for ifenprodil. The overall fold of our GluN1-NTD crystal structure was similar to that of the GluN2B-NTD, and thus if the binding cleft of GluN1NTD is solvent accessible it is conceivable that some unidentified ligand may potentially bind to the GluN1-NTD cleft. Molecules that bind to the pockets of NTDs of the NMDA-R can potentially modulate the channel gating (Gielen et al., 2009) and also the subunit assembly by influencing the heterodimer interface of GluN1-NTD and GluN2-NTD. It would now be time to initiate the search for putative binding molecules and their function.

\section{References}

Abrahams JP, Leslie AG (1996) Methods used in the structure determination of bovine mitochondrial F-1 ATPase. Acta Crystallogr D 52:30-42.

Andersson O, Stenqvist A, Attersand A, von Euler G (2001) Nucleotide sequence, genomic organization, and chromosomal localization of genes encoding the human NMDA receptor subunits NR3A and NR3B. Genomics 78:178-184.

Atlason PT, Garside ML, Meddows E, Whiting P, McIlhinney RA (2007) N-Methyl-D-aspartate (NMDA) receptor subunit NR1 forms the substrate for oligomeric assembly of the NMDA receptor. J Biol Chem 282:25299-25307.

Ayalon G, Stern-Bach Y (2001) Functional assembly of AMPA and kainate receptors is mediated by several discrete protein-protein interactions. Neuron 31:103-113.

Chen C, Okayama H (1987) High-efficiency transformation of mammalian cells by plasmid DNA. Mol Cell Biol 7:2745-2752.

Choi YB, Lipton SA (1999) Identification and mechanism of action of two histidine residues underlying high-affinity $\mathrm{Zn}^{2+}$ inhibition of the NMDA receptor. Neuron 23:171-180.

Clayton A, Siebold C, Gilbert RJ, Sutton GC, Harlos K, McIlhinney RA, Jones EY, Aricescu AR (2009) Crystal structure of the GluR2 amino-terminal domain provides insights into the architecture and assembly of ionotropic glutamate receptors. J Mol Biol 392:1125-1132.

Collaborative Computational Project, Number 4 (1994) The CCP4 suite: programs for protein crystallography. Acta Crystallogr D Biol Crystallogr 50:760-763.

Cull-Candy SG, Leszkiewicz DN (2004) Role of distinct NMDA receptor subtypes at central synapses. Sci STKE 2004:re16.

de La Fortelle E, Bricogne G (1997) Maximum-likelihood heavy-atom parameter refinement for multiple isomorphous replacement and multiwavelength anomalous diffraction methods. In: Methods in enzymology, Vol 276, Chap 7 (Carter CW, Sweet RM, eds), pp 472-494. New York: Academic.

Dingledine R, Borges K, Bowie D, Traynelis SF (1999) The glutamate receptor ion channels. Pharmacol Rev 51:7-61.

Emsley P, Lohkamp B, Scott WG, Cowtan K (2010) Features and development of Coot. Acta Crystallogr D Biol Crystallogr 66:486-501.

Forrest D, Yuzaki M, Soares HD, Ng L, Luk DC, Sheng M, Stewart CL, Morgan JI, Connor JA, Curran T (1994) Targeted disruption of NMDA receptor 1 gene abolishes NMDA response and results in neonatal death. Neuron 13:325-338.

Frank J, Radermacher M, Penczek P, Zhu J, Li Y, Ladjadj M, Leith A (1996) SPIDER and WEB: processing and visualization of images in $3 \mathrm{D}$ electron microscopy and related fields. J Struct Biol 116:190-199. 
Furukawa H, Singh SK, Mancusso R, Gouaux E (2005) Subunit arrangement and function in NMDA receptors. Nature 438:185-192.

Gielen M, Le Goff A, Stroebel D, Johnson JW, Neyton J, Paoletti P (2008) Structural rearrangements of NR1/NR2A NMDA receptors during allosteric inhibition. Neuron 57:80-93.

Gielen M, Siegler Retchless B, Mony L, Johnson JW, Paoletti P (2009) Mechanism of differential control of NMDA receptor activity by NR2 subunits. Nature 459:703-707.

Greger IH, Ziff EB, Penn AC (2007) Molecular determinants of AMPA receptor subunit assembly. Trends Neurosci 30:407-416.

Ho BK, Gruswitz F (2008) HOLLOW: generating accurate representations of channel and interior surfaces in molecular structures. BMC Struct Biol 8:49.

Hollmann M, Maron C, Heinemann S (1994) N-glycosylation site tagging suggests a three transmembrane domain topology for the glutamate receptor GluR1. Neuron 13:1331-1343.

Inanobe A, Furukawa H, Gouaux E (2005) Mechanism of partial agonist action at the NR1 subunit of NMDA receptors. Neuron 47:71-84.

Izumi Y, Auberson YP, Zorumski CF (2006) Zinc modulates bidirectional hippocampal plasticity by effects on NMDA receptors. J Neurosci 26:7181-7188.

Jin R, Singh SK, Gu S, Furukawa H, Sobolevsky AI, Zhou J, Jin Y, Gouaux E (2009) Crystal structure and association behaviour of the GluR2 aminoterminal domain. EMBO J 28:1812-1823.

Karakas E, Simorowski N, Furukawa H (2009) Structure of the zinc-bound amino-terminal domain of the NMDA receptor NR2B subunit. EMBO J 28:3910-3920.

Kemp JA, McKernan RM (2002) NMDA receptor pathways as drug targets. Nat Neurosci [Suppl] 5:1039-1042.

Kumar J, Mayer ML (2010) Crystal structures of the glutamate receptor ion channel GluK3 and GluK5 amino-terminal domains. J Mol Biol 404:680-696.

Kumar J, Schuck P, Jin R, Mayer ML (2009) The N-terminal domain of GluR6-subtype glutamate receptor ion channels. Nat Struct Mol Biol 16:631-638.

Kunishima N, Shimada Y, Tsuji Y, Sato T, Yamamoto M, Kumasaka T, Nakanishi S, Jingami H, Morikawa K (2000) Structural basis of glutamate recognition by a dimeric metabotropic glutamate receptor. Nature 407:971-977.

Kuusinen A, Abele R, Madden DR, Keinänen K (1999) Oligomerization and ligand-binding properties of the ectodomain of the alpha-amino-3hydroxy-5-methyl-4-isoxazole propionic acid receptor subunit GluRD. J Biol Chem 274:28937-28943.

Laskowski RA, MacArthur MW, Moss DS, Thornton JM (1993) PROCHECKa program to check the stereochemical quality of protein structures. J Appl Crystallogr 26:283-291.

Leuschner WD, Hoch W (1999) Subtype-specific assembly of alpha-amino3-hydroxy-5-methyl-4-isoxazole propionic acid receptor subunits is mediated by their N-terminal domains. J Biol Chem 274:16907-16916.

Low CM, Zheng F, Lyuboslavsky P, Traynelis SF (2000) Molecular determinants of coordinated proton and zinc inhibition of $\mathrm{N}$-methyl-D-aspartate NR1/NR2A receptors. Proc Natl Acad Sci U S A 97:11062-11067.

McIlhinney RA, Le Bourdellès B, Molnár E, Tricaud N, Streit P, Whiting PJ (1998) Assembly intracellular targeting and cell surface expression of the human $N$-methyl-D-aspartate receptor subunits NR1a and NR2A in transfected cells. Neuropharmacology 37:1355-1367.

Meddows E, Le Bourdelles B, Grimwood S, Wafford K, Sandhu S, Whiting P, McIlhinney RA (2001) Identification of molecular determinants that are important in the assembly of $\mathrm{N}$-methyl-D-aspartate receptors. J Biol Chem 276:18795-18803.

Monyer H, Sprengel R, Schoepfer R, Herb A, Higuchi M, Lomeli H, Burnashev N, Sakmann B, Seeburg PH (1992) Heteromeric NMDA receptors: molecular and functional distinction of subtypes. Science 256:1217-1221.

Moriyoshi K, Masu M, Ishii T, Shigemoto R, Mizuno N, Nakanishi S (1991)
Molecular cloning and characterization of the rat NMDA receptor. Nature 354:31-37.

Murshudov GN, Vagin AA, Dodson EJ (1997) Refinement of macromolecular structures by the maximum-likelihood method. Acta Crystallogr D Biol Crystallogr 53:240-255.

Nakagawa T, Cheng Y, Ramm E, Sheng M, Walz T (2005) Structure and different conformational states of native AMPA receptor complexes. Nature 433:545-549.

Ohi M, Li Y, Cheng Y, Walz T (2004) Negative staining and image classification - powerful tools in modern electron microscopy. Biol Proced Online 6:23-34.

Otwinowski Z, Minor W (1997) Processing of X-ray diffraction data collected in oscillation mode. Method Enzymol 276:307-326.

Palay SL, Palade GE (1955) The fine structure of neurons. J Biophys Biochem Cytol 1:69-88.

Paoletti P, Perin-Dureau F, Fayyazuddin A, Le Goff A, Callebaut I, Neyton J (2000) Molecular organization of a zinc binding N-terminal modulatory domain in a NMDA receptor subunit. Neuron 28:911-925.

Perin-Dureau F, Rachline J, Neyton J, Paoletti P (2002) Mapping the binding site of the neuroprotectant ifenprodil on NMDA receptors. J Neurosci 22:5955-5965.

Peters A, Palay SL, Webster Hd (1991) The fine structure of the nervous system: neurons and their supporting cells, 3rd Ed. New York: Oxford UP.

Qiu S, Hua YL, Yang F, Chen YZ, Luo JH (2005) Subunit assembly of $\mathrm{N}$-methyl-D-aspartate receptors analyzed by fluorescence resonance energy transfer. J Biol Chem 280:24923-24930.

Qiu S, Zhang XM, Cao JY, Yang W, Yan YG, Shan L, Zheng J, Luo JH (2009) An endoplasmic reticulum retention signal located in the extracellular amino-terminal domain of the NR2A subunit of $N$-methyl-D-aspartate receptors. J Biol Chem 284:20285-20298.

Rachline J, Perin-Dureau F, Le Goff A, Neyton J, Paoletti P (2005) The micromolar zinc-binding domain on the NMDA receptor subunit NR2B. J Neurosci 25:308-317.

Reeves PJ, Callewaert N, Contreras R, Khorana HG (2002) Structure and function in rhodopsin: high-level expression of rhodopsin with restricted and homogeneous $N$-glycosylation by a tetracycline-inducible $\mathrm{N}$-acetylglucosaminyltransferase I-negative HEK293S stable mammalian cell line. Proc Natl Acad Sci U S A 99:13419-13424.

Scannevin RH, Huganir RL (2000) Postsynaptic organization and regulation of excitatory synapses. Nat Rev Neurosci 1:133-141.

Schneider TR, Sheldrick GM (2002) Substructure solution with SHELXD. Crystallogr D Biol Crystallogr 58:1772-1779.

Schüler T, Mesic I, Madry C, Bartholomäus I, Laube B (2008) Formation of NR1/NR2 and NR1/NR3 heterodimers constitutes the initial step in $N$-methyl-D-aspartate receptor assembly. J Biol Chem 283:37-46.

Shanks NF, Maruo T, Farina AN, Ellisman MH, Nakagawa T (2010) Contribution of the global subunit structure and stargazin on the maturation of AMPA receptors. J Neurosci 30:2728-2740.

Sobolevsky AI, Rosconi MP, Gouaux E (2009) X-ray structure, symmetry and mechanism of an AMPA-subtype glutamate receptor. Nature 462:745-756.

Stroebel D, Carvalho S, Paoletti P (2011) Functional evidence for a twisted conformation of the NMDA receptor GluN2A subunit N-terminal domain. Neuropharmacology 60:151-158.

Sugihara H, Moriyoshi K, Ishii T, Masu M, Nakanishi S (1992) Structures and properties of seven isoforms of the NMDA receptor generated by alternative splicing. Biochem Biophys Res Commun 185:826-832.

Traynelis SF, Wollmuth LP, McBain CJ, Menniti FS, Vance KM, Ogden KK, Hansen KB, Yuan H, Myers SJ, Dingledine R (2010) Glutamate receptor ion channels: structure, regulation, and function. Pharmacol Rev 62:405-496.

Vonrhein C, Blanc E, Roversi P, Bricogne G (2007) Automated structure solution with autoSHARP. Methods Mol Biol 364:215-230. 